OPEN ACCESS

Edited by:

Francesca Patrignani,

Università degli Studi di Bologna, Italy

Reviewed by:

Giulia Tabanelli,

Università degli Studi di Bologna, Italy Carmen Wacher,

Universidad Nacional Autónoma de México, Mexico

*Correspondence: Emmanuel Coton emmanuel.coton@univ-brest.fr

Specialty section: This article was submitted to Food Microbiology,

a section of the journal

Frontiers in Microbiology

Received: 20 April 2018

Accepted: 17 July 2018 Published: 07 August 2018

Citation:

Leyva Salas M, Thierry A, Lemaitre $M$, Garric G, Harel-Oger M, Chatel M,

Lê S, Mounier J, Valence F and Coton E (2018) Antifungal Activity of Lactic Acid Bacteria Combinations in Dairy Mimicking Models and Their

Potential as Bioprotective Cultures in Pilot Scale Applications.

Front. Microbiol. 9:1787.

doi: 10.3389/fmicb.2018.01787

\section{Antifungal Activity of Lactic Acid Bacteria Combinations in Dairy Mimicking Models and Their Potential as Bioprotective Cultures in Pilot Scale Applications}

\author{
Marcia Leyva Salas ${ }^{1,2}$, Anne Thierry $^{2}$, Mathilde Lemaître ${ }^{1}$, Gilles Garric ${ }^{2}$, \\ Marielle Harel-Oger ${ }^{2}$, Manon Chatel ${ }^{2}$, Sébastien Lê ${ }^{3}$, Jérôme Mounier ${ }^{1}$, \\ Florence Valence ${ }^{2}$ and Emmanuel Coton ${ }^{1 *}$ \\ ${ }^{1}$ Université de Brest, EA 3882 Laboratoire Universitaire de Biodiversité et Ecologie Microbienne, ESIAB, Technopôle \\ Brest-Iroise, Plouzané, France, ${ }^{2}$ UMR1253 Science et Technologie du Lait et de l'CEuf, INRA, Agrocampus Ouest, Rennes, \\ France, ${ }^{3}$ Applied Mathematics Department, Agrocampus Ouest, Rennes, France
}

Consumer's demand for naturally preserved food products is growing and the use of bioprotective cultures is an alternative to chemical preservatives or a complementary tool to hurdle technologies to avoid or delay fungal spoilage of dairy products. To develop antifungal cultures for the dairy product biopreservation, experiments were conducted both in vitro and in situ. Firstly, the antifungal activity of 32 strains of lactic acid bacteria $(\llcorner\mathrm{AB})$ and propionibacteria was screened alone, and then on combinations based on 5 selected lactobacilli strains. This screening was performed in yogurt and cheese models against four major spoilage fungi previously isolated from contaminated dairy products (Penicillium commune, Mucor racemosus, Galactomyces geotrichum, and Yarrowia lipolytica). Selected combinations were then tested as adjunct cultures in sour cream and semi-hard cheeses produced at a pilot scale to evaluate their antifungal activity during challenge tests against selected fungal targets ( $P$. commune, $M$. racemosus, and Rhodotorula mucilaginosa) and shelf life tests; and their impact on product organoleptic properties. The screening step allowed selecting two binary combinations, A1 and A3 composed of Lactobacillus plantarum L244 and either Lactobacillus harbinensis L172 or Lactobacillus rhamnosus CIRM-BIA1113, respectively. In situ assays showed that the A1 combination delayed the growth of $P$. commune, $M$. racemosus and $R$. mucilaginosa for 2-24 days on sour cream depending of the antifungal culture inoculum, without effect on organoleptic properties at low inoculum ( $10^{6}$ colony-forming units (CFU)/mL). Moreover, the $\mathrm{A} 1$ and $\mathrm{A} 3 \mathrm{combinations}$ also delayed the growth of $P$. commune in semi-hard cheese for 1-6 days and 1 day, respectively. Antifungal cultures neither impacted the growth of starter cultures in both sour cream and cheese nor the products' $\mathrm{pH}$, although post acidification was observed in sour cream supplemented with these combinations at 
the highest concentrations $\left(2.10^{7} \mathrm{CFU} / \mathrm{mL}\right)$. The combination of both in vitro and in situ screening assays allowed developing 2 antifungal combinations exhibiting significant antifungal activity and providing future prospects for use as bioprotective cultures in dairy products.

Keywords: biopreservation, dairy products, propionibacteria, Lactobacillus harbinensis, L. plantarum, L. rhamnosus

\section{INTRODUCTION}

Food spoilage by fungi (molds or yeasts) is responsible for considerable food waste and economical losses. Among food products, fermented dairy products can be affected by fungal spoilers resistant to low $\mathrm{pH}$ and able to grow at low storage temperatures $\left(<10^{\circ} \mathrm{C}\right)$. These contaminants exhibit proteolytic and lipolytic capacities, and consume the dairy product main sugars and organic acids (lactic and citric acids), thus impacting the product organoleptic qualities (Pitt and Hocking, 2009). Fungal spoilers can affect the product aspect (presence of thallus or yeast colonies, gas production), texture, odor, and flavor (Ledenbach and Marshall, 2009; Pitt and Hocking, 2009). In this context, the main fungal genera encountered in contaminated dairy products and production environments correspond to Penicillium, Mucor, and Cladosporium for molds, and Candida, Meyerozyma, and Yarrowia for yeasts (Pitt and Hocking, 2009; Garnier et al., 2016).

Preclusion of fungal spoilage in food currently relies on prevention methods (e.g., implementation of Hazard Analysis Critical Point -HACCP-), hurdle technologies (e.g., heat treatments, water removal, modified atmosphere packaging, salting, fermentation) and the use of chemical preservatives, including benzoate, propionate, sorbate, nitrate, and sulfites (Silva and Lidon, 2016). For fungal control in dairy products, potassium sorbate and natamycin (E235), a microbial food preservative produced by Streptomyces natalensis belonging to the group of polyene macrolide antimycotics, are the main preservatives used. Natamycin is approved by most countries for use as a cheese surface treatment while its addition into other foods and the permitted applications differ per country/region (Stark and Tan, 2003). Agri-food industries rely mainly on chemical preservatives to control microbial contaminant growth and extend the product shelf life, but a strong societal demand for less processed and preservative-free food has emerged, and additive regulations are constantly evolving to limit their use (Fuselli et al., 2012). The latter aspects, combined with the necessity to produce food products complying with high safety and quality standards, have led to the search for alternatives to the use of chemical preservatives. Among the explored natural alternatives, biopreservation, corresponding to use of microorganisms and/or their antimicrobial metabolites (Stiles, 1996), has recently attract much interest.

In the dairy product context, lactic acid bacteria (LAB) and propionibacteria $(\mathrm{PAB})$, which are naturally present or used as cultures in these matrices, play a key role in the fermentation process. Fermentation is a "natural" preservation technique that improves food safety, nutritional value and specific organoleptic features (e.g., eye formation in Swisstype cheeses by propionibacteria) (Bourdichon et al., 2012; Fröhlich-Wyder et al., 2017). Bacterial fermentation induces acidification, due to the production of organic acids (e.g., lactic, acetic, or propionic acids), which play a role in fermented food biopreservation (Batish et al., 1997; Leroy and De Vuyst, 2004). These organic acids as well as other metabolites such as phenyllactic, hydroxyphenyllactic, benzoic acids, fatty acids, volatile compounds (e.g., diacetyl, acetoin), cyclic dipeptides, hydrogen peroxide, reuterin, and/or proteinaceous compounds have exhibited an antifungal activity (Crowley et al., 2013; Leyva Salas et al., 2017). The use of LAB and PAB to reduce levels or fully replace antifungal chemical preservatives is clearly an alternative of interest as these microorganisms are recognized as safe (as confirmed by their Qualified Presumption of Safety-QPSor Generally Recognize As Safe -GRAS- status), are known to have a long history of safe use and obviously exhibit antifungal properties (Bourdichon et al., 2012). In this context, several studies of $\mathrm{LAB}$ and $\mathrm{PAB}$ as bioprotective cultures with antifungal activity have been reported during the last years in various dairy products (yogurt, semi-hard ewe milk cheese, cottage cheese, and cheddar cheese; Delavenne et al., 2013, 2015; Li et al., 2013; Cheong et al., 2014; Lynch et al., 2014; Aunsbjerg et al., 2015; Gómez-Torres et al., 2016; Fernandez et al., 2017) Noteworthy, despite the number of reported studies, only a few number of antifungal cultures are available on the market due to several constraints before commercialization of a strain of interest (e.g., gap between in vitro and in situ activity, undesirable organoleptic impacts on the products, safety evaluation and cell viability) (Leyva Salas et al., 2017).

The aim of this study was to develop bioprotective cultures with antifungal activity suitable for use in the dairy industry as adjunct cultures. To do so, a scale-up approach, going from antifungal activity screening in dairy models to pilot scale applications in actual dairy products, was performed. Several aspects were considered in order to get close to actual dairy industry conditions: (i) antifungal activity screening of 32 strains compatible with a use as adjunct cultures, (ii) use of two dairy models for the in vitro tests, (iii) antifungal activity test against fungal targets isolated from contaminated dairy products, (iv) assessment of the antifungal activity of bioprotective cultures at a pilot scale in two dairy products, and (v) evaluation of the impact of the antifungal bioprotective cultures on the starter cultures as well as the $\mathrm{pH}$ and organoleptic traits of the final products. 


\section{MATERIALS AND METHODS}

\section{Microorganisms and Culture Conditions}

LAB (23 strains) and PAB (9 strains) (Table 1) were obtained from the Université de Bretagne Occidentale (UBOCC, France) and CIRM-BIA (INRA, France) culture collections. They all corresponded to strains that were previously shown to exhibit antifungal activity, either directly (Delavenne et al., 2012) or indirectly (use of fermentates, i.e., ingredients produced by the fermentation of a dairy matrices) (Garnier et al., 2018). These strains, presenting diversity at the species (19 Lactobacillus, 4 Leuconostoc, and 9 Propionibacterium) and intraspecific level, were selected based on their compatibility with dairy technology. Moreover, three strains isolated on de Man-Rogosa-Sharpe (MRS) agar from commercial bioprotective cultures were used as reference antifungal strains in yogurt, cheese model and sour cream. They corresponded to Lactobacillus rhamnosus CIRM-BIA1759 and Lactobacillus paracasei CIRM-BIA1761 isolated from the commercial culture CC1, while Lactobacillus plantarum CIRM-BIA1758 was isolated from the commercial culture CC2. Culture X1 corresponded to a 1:1 combination of the L. paracasei CIRM-BIA1759 and L. rhamnosus CIRMBIA1761 strains, while X2 corresponded to L. plantarum CIRMBIA1758 alone. For semi-hard cheese production, a commercial culture CC3 (consisting of the same species and purchased from the same supplier that CC1) was directly applied as recommended by the supplier. All strains were stored at $-80^{\circ} \mathrm{C}$ in MRS broth (BD Difco laboratories, Sparks, MD, USA) supplemented with glycerol (20\%) (Thermo Fischer Scientific, Waltham, MA, USA). Before inoculation as adjunct cultures, strains were cultivated twice in MRS broth for $\mathrm{LAB}$ and in yeast extract lactate (YEL) medium for $\mathrm{PAB}$ at $30^{\circ} \mathrm{C}$ for 24 and $48 \mathrm{~h}$, respectively. Culture concentration was then adjusted to the target concentrations with sodium chloride peptone broth (Merck, Darmstadt, Germany). After centrifugation, the cells were resuspended in sterile skimmed milk. Various LAB commercial starters (i.e., used for their technological properties) were used for screening tests and pilot scale experiments. They corresponded to MA016 (Lactococcus lactis subsp. cremoris and L. lactis subsp. lactis, Elimeca, Thoissey, France); MM100 (L. lactis subsp. lactis, L. lactis subsp. cremoris, and L. lactis subsp. lactis biovar diacetylactis, Choozit, Danisco, Sassenage, France); MD88 (L. lactis subsp. lactis biovar diacetylactis, Choozit, Danisco, Sassenage, France); MY800 (Streptococcus thermophilus, Lactobacillus delbrueckii subsp. lactis and L. delbrueckii subsp. bulgaricus, Choozit, Danisco, Sassenage, France) and PAL YOG 1-30D (S. thermophilus and Lactobacillus delbrueckii subsp. bulgaricus, Standa, Caen, France).

The 10 fungal targets used, 4 filamentous molds and 6 yeasts, were from the UBOCC collection (Plouzané, France). They corresponded to Candida parapsilosis UBOCC-A-216002, Galactomyces geotrichum UBOCC-A-216001, Meyerozyma guilliermondii UBOCC-A-216003, Mucor racemosus UBOCCA-116002, Penicillium commune UBOCC-A-116003, Penicillium bialowiezense UBOCC-A-117365, Didymella pinodella UBOCCA-116004, Yarrowia lipolytica UBOCC-A-216006, Rhodotorula mucilaginosa UBOCC-A-216004 and Trichosporon asahii
UBOCC-A-216005. Filamentous fungi were cultivated and then stored at $-80^{\circ} \mathrm{C}$ as a spore or cell suspension in sterile glycerol as previously described (Delavenne et al., 2012), and yeasts were pre-cultivated in potato dextrose broth (PDB, Difco, Becton Dickinson, Sparks, MD, USA). Spore or cell concentrations in suspension were determined using a haemocytometer (Malassez, Preciss, Paris, France) and adjusted with sodium chloride peptone broth to $5.10^{3}$ spores or cells/mL.

\section{Antifungal Activity Screening of Bacterial Strains}

Antifungal activity was screened using the high-throughput method developed by Garnier et al. (2018) on two dairy matrices: the same cheese-mimicking matrix used by the latter authors and a yogurt. The cheese-mimicking matrix (hereafter named model cheese) was prepared from a heat-treated salted $0.7 \%$ $(\mathrm{w} / \mathrm{w})$ ultrafiltered milk retentate, kept at $-20^{\circ} \mathrm{C}$ until use. The model cheese was defrosted at $48^{\circ} \mathrm{C}$ for $3 \mathrm{~min}$, then $10 \mathrm{~mL} / \mathrm{L}$ of a $\mathrm{pH}$ indicator (sterile solution of Litmus $50 \mathrm{~g} / \mathrm{L}$ ), $10^{6}$ colony forming unit (CFU)/mL of commercial starters (either MA016, MM100 or MD88) and $1.5 \mathrm{~mL} / \mathrm{L}$ of $5 \mathrm{X}$ diluted and filtered $(0.22 \mu \mathrm{m})$ chymosin rennet (Maxiren 180, DSM Food Specialties, The Netherlands) were added to the thawed model cheese. After vigorous homogenization for $1 \mathrm{~min}$, it was distributed into 24well plates $(2 \mathrm{~mL} /$ well), cell suspensions in milk of the tested strains were added or not (positive and negative fungal growth control wells) at $10 \% \mathrm{v} / \mathrm{v}$ at a final concentration of $10^{5}$ and $10^{7}$ $\mathrm{CFU} / \mathrm{mL}$ ( 2 wells per concentration and 1 strain by column) and plates were incubated for $1 \mathrm{~h}$ at $30^{\circ} \mathrm{C}$ then for 3 days at $20^{\circ} \mathrm{C}$ with a final $\mathrm{pH}$ of $4.8 \pm 0.1$. The screening method was also adapted to yogurt. To do so, yogurt was prepared from semiskimmed milk (Candia, France) supplemented with skimmed milk powder at $4 \%$ (Carrefour, France). After heat treatment for $30 \mathrm{~min}$ at $85^{\circ} \mathrm{C}$, milk was rapidly cooled to $45^{\circ} \mathrm{C}$ before adding $0.1 \mathrm{~g} / \mathrm{L}$ of the lyophilized starter culture (either MY800 or PAL YOG 1-30D) and $\mathrm{pH}$ indicator. After homogenization, the preparation was distributed into 24 -well plates $(2 \mathrm{~mL} /$ well $)$ and the antifungal strain suspensions were added under the same conditions as for the model cheese. Plates were then incubated at $42^{\circ} \mathrm{C}$ during 4 or $6 \mathrm{~h}$ (for starters MY800 and PAL-YOG$1-30 \mathrm{D}$, respectively) until $\mathrm{pH} 4.97 \pm 0.03$ was reached. For the two dairy models, at the end of fermentation, exudate was removed and 50 spores/cells of either $M$. racemosus, $P$. commune, $Y$. lipolytica, or G. geotrichum were spotted at the center of each well (1 fungi tested/plate) except for the positive control wells. Antifungal activity was assessed by visually evaluating fungal growth in comparison with the negative control, after incubation for up to 8 days at 12 and $10^{\circ} \mathrm{C}$ (for cheese and yogurt plates, respectively). Antifungal activity scoring was done using a qualitative system presented in Figure $\mathbf{1}$ and by recording the days of total inhibition until fungal growth was visible. Moreover, a global score was calculated for each tested culture at a given inoculum. This global score corresponded to the sum of a numeric equivalent of the antifungal score ( 0 , equivalent to - , corresponded to no inhibition and 3 , equivalent to +++ , 


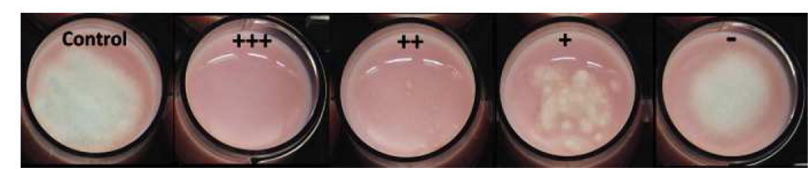

FIGURE 1 | Example of the antifungal inhibition scoring in yogurt against $P$. commune. Control, yogurt with no antifungal culture; +++ , total inhibition; ++ , slight inhibition; +, very low inhibition; -, no inhibition.

corresponded to total inhibition) divided by the total number of tested fungal targets.

A first antifungal activity screening was performed for the 32 selected strains in the two dairy models with only one commercial starter per model (MY800 and MA016 for yogurt and cheese, respectively). In a second step, the impact of the commercial starters on the observed antifungal activity of 12 strains was evaluated using 3 other commercial starters. Then, 5 strains of interest were selected to be evaluated in combinations.

\section{Evaluation of the Antifungal Activity of Strain Combinations}

Ten binary and three ternary combinations, based on a selection of 5 strains, were tested using the same screening method on yogurt and model cheese with only one starter for each model, and compared to the individual strains to evaluate combination effects. Combinations of interest were then tested for antifungal activity at four inoculum levels ranging from $10^{6}$ to $2.10^{7} \mathrm{CFU} / \mathrm{mL}$ in the two models in the presence of different commercial starters. Then, to evaluate the action spectrum of the selected antifungal combinations and pure cultures, six supplementary fungal targets, namely $D$. pinodella, $C$. parapsilosis, M. guilliermondii, P. bialowiezense, R. mucilaginosa, and T. asahii, were studied in yogurt and cheese models.

\section{Antifungal Activity in Pilot Scale Conditions}

Antifungal activity of the selected combinations was then determined in two types of dairy products, sour cream and semihard cheese, produced at a pilot scale at the Dairy Platform of INRA Rennes, France.

\section{Challenge-Tests in Sour Cream}

Sour cream (final fat $35 \%$ ) was produced from $25 \mathrm{~L}$ of pasteurized $\left(20 \mathrm{~s}\right.$ at $\left.75^{\circ} \mathrm{C}\right)$, skimmed and standardized cow milk $\left(50^{\circ} \mathrm{C}, 35 \%\right.$ fat). Skimmed milk was then homogenized $\left(65^{\circ} \mathrm{C}, 50\right.$ bars, and then 5 bars) and inoculated with a commercial starter (MM100) at $3 \mathrm{U} / 100 \mathrm{~L}$, the selected antifungal combinations (either $10^{6}$, $5.10^{6}$ or $\left.2.10^{7} \mathrm{CFU} / \mathrm{mL}\right)$ or the $\mathrm{X} 1$ combination $\left(10^{6} \mathrm{CFU} / \mathrm{mL}\right)$ before packaging in $100 \mathrm{~g}$ plastic tubs with mobile lids. After overnight incubation $\left(22 \mathrm{~h}\right.$ at $\left.23^{\circ} \mathrm{C}\right)$, sour cream samples were challenged. For positive controls, instead of the bioprotective cultures, potassium sorbate (Sigma-Aldrich, Saint-Louis, MO, USA) was added at $0.08 \%$ after acidification and mixed using a sterile spatula. Negative control corresponded to the sour cream containing only the technological starter. For challenge-tests, 50 fungal spores of either P. commune UBOCC-A-116003 or
M. racemosus UBOCC-A-116002 were inoculated on the sour cream surface and incubated at $10^{\circ} \mathrm{C}$. Fungal growth was then visually evaluated daily during 4 weeks. For a third fungal target, R. mucilaginosa UBOCC-A-216004, cream tubs were inoculated at $2 \mathrm{CFU} / \mathrm{g}$ and mixed with a sterile spatula. Yeast growth was evaluated by enumeration on YEGC medium, containing 20 g/L of glucose (Sigma-Aldrich, Saint-Louis, MO, USA), 5 g/L of yeast extract (Merck, Darmstadt, Germany) and $0.5 \mathrm{~g} / \mathrm{L}$ of chloramphenicol (Sigma-Aldrich, Saint-Louis, MO, USA) after incubation for 7 days at $10^{\circ} \mathrm{C}$. A number of non-challenged cream tubs were stored at $4^{\circ} \mathrm{C}$ for bacterial control, $\mathrm{pH}$ control, shelf life test and sensory analyses.

\section{Challenge-Tests in Semi-hard Cheese}

Two replicate cheese trials were undertaken on two separate weeks. For each trial, four vats of cheese were produced, one for each antifungal combination and one control cheese without antifungal culture. Cheese were manufactured as follows. Cow milk was pasteurized at $72^{\circ} \mathrm{C}$ for $20 \mathrm{~s}$, standardized to $30 \mathrm{~g}$ fat $/ \mathrm{kg}$ milk, added with $36 \mathrm{mg}$ Ca per $\mathrm{kg}(20 \mathrm{~mL}$ per $100 \mathrm{~kg}$ milk of a $500 \mathrm{~g} / \mathrm{L} \mathrm{CaCl} 2$ solution), then cooled to $4^{\circ} \mathrm{C}$ and stored overnight. Prior to cheese making, the milk was pumped into $30 \mathrm{~L}$ cylindrical jacketed stainless steel cheese vats, with variable speed cutting and stirring (Frominox, Assat, France) and warmed to $33^{\circ} \mathrm{C}$. The MA016 commercial starter, previously suspended in UHT semi-skimmed milk and let to rehydrate for $1 \mathrm{~h}$ at $20^{\circ} \mathrm{C}$, was added to the cheese milk at $0.5 \mathrm{U} / 100 \mathrm{~kg}$ (i.e., $\sim 10^{6} \mathrm{CFU} / \mathrm{mL}$ ). Antifungal cultures were added at $5.10^{6}$ $\mathrm{CFU} / \mathrm{mL}$ (each strain at 2.5.10 $\mathrm{CFU} / \mathrm{mL}$ for the $\mathrm{A} 1$ and $\mathrm{A} 3$ combinations, and the commercial culture CC3). After around $30 \mathrm{~min}$ pre-ripening at $33^{\circ} \mathrm{C}(\mathrm{pH} 6.57-6.59), 0.25 \mathrm{~mL} / \mathrm{kg}$ of animal rennet $(520 \mathrm{mg} / \mathrm{L}$ chymosin, Carlina 145/80, Dupont Danisco, Dangé, Saint Romain, France) diluted in deionized water was added. After coagulation (34-37 min), curd was cut to the size of corn grains, washed with warm water, cooked for $20 \mathrm{~min}$ at $35^{\circ} \mathrm{C}$, and drained. Curd was pre-pressed at $60 \mathrm{kPa}$ for $30 \mathrm{~min}$, molded (700 g curd per mold), and pressed at $300 \mathrm{kPa}$ for $30 \mathrm{~min}$ followed by $600 \mathrm{kPa}$ for $90 \mathrm{~min}$. The obtained cheeses remained in the mold overnight, the temperature decreased from $\sim 25$ to $\sim 20^{\circ} \mathrm{C}$. At demolding, cheeses had a pH of $5.11 \pm 0.04$ and contained $51.41 \pm 1.13 \%$ dry matter $(\mathrm{pH}$ of $5.14 \pm 0.03$ and $5.09 \pm 0.04$ and dry matter of $50.79 \pm 0.75$, and $52.11 \pm$ 1.02 , for cheese trials 1 and 2, respectively) and 45\% fat in dry matter. Cheeses were salted by immersion at $20^{\circ} \mathrm{C}$ for $4 \mathrm{~h}$ in a sterile brine containing $33 \%(\mathrm{~m} / \mathrm{m}) \mathrm{NaCl}, 36 \mathrm{mg} / \mathrm{L} \mathrm{Ca}, \mathrm{pH}$ 5.2 , and were let to dry overnight at room temperature (20$25^{\circ} \mathrm{C}$ ) under a laminar flow hood. The external part of cheeses ( $3 \mathrm{~mm}$ thickness) was then sterilely discarded and their surface smeared with a smear preparation containing the commercial cultures Debaryomyces hansenii CHOOZIT DH, Kluyveromyces lactis CHOOZIT KL71, and Brevibacterium linens CHOOZIT SR3, (Danisco, Dangé Saint-Romain, France) at $0.01 \% \mathrm{w} / \mathrm{w}$ each in a sterile $0.1 \% \mathrm{NaCl}$ solution, $\mathrm{pH} 5.2$. Cheeses were then dried for $1 \mathrm{~h}$ at room temperature under laminar flow hood and then transferred to ripening chambers $\left(12^{\circ} \mathrm{C}\right)$. After 1 day in the ripening chambers, the upper cheese surface was inoculated with three spots of 50 spores of either P. commune 
or $M$. racemosus as fungal targets. For positive controls, instead of bioprotective cultures, natamycin (LCP natose, Laboratoires Humeau, France) was applied by spray over the upper surface of the cheese at a final concentration of $0.5 \mathrm{mg} / \mathrm{dm}^{2}$ before spore inoculation. Some cheeses without spore inoculation were also included for microbial, biochemical analyses, shelf life tests and sensory evaluations. All cheeses were ripened for four weeks at $12^{\circ} \mathrm{C}$ and $96 \%$ relative humidity (1 chamber per fungal target). Cheeses were sampled aseptically for $\mathrm{pH}$ measurement, microbial analyses of starters and antifungal cultures over a period of 4 weeks at 1 week intervals.

\section{Bacterial Growth and Physicochemical Parameters}

To evaluate the growth of antifungal strains and their potential impact on the commercial lactic starters, bacterial populations were enumerated before and after fermentation as well as during storage (once a week). To do so, sour cream (1 g) samples were diluted in sodium chloride peptone broth and semi-hard cheese $(10 \mathrm{~g})$ samples were blended in sterile bags with $2 \%$ trisodium citrate (Sigma-Aldrich, Saint-Louis, MO, USA) using a stomacher (Merck Eurolab, Strasbourg, France). Antifungal combinations were cultivated on LAMVAB agar (Hartemink et al., 1997) and incubated under anaerobic conditions (Anaerocult A; Merck, Darmstadt, Germany) at $30^{\circ} \mathrm{C}$ for 5 days. MM100 and MA016 starters were cultivated on M17 agar (Difco Laboratories, USA) supplemented with $0.5 \%$ lactose and incubated for $24 \mathrm{~h}$ at $30^{\circ} \mathrm{C}$ in aerobic conditions.

\section{Shelf Life Tests}

Shelf life tests were performed to evaluate the antifungal activity exerted by the selected associations in the produced sour cream and semi-hard cheeses (free from fungal targets), in a naturally contaminated environment. For sour cream, after 2 weeks of storage at $4^{\circ} \mathrm{C}, 200 \mathrm{~g}$ of each condition were spread out in ten $100 \mathrm{~mm}$ Petri dishes. The plates were then exposed for $20 \mathrm{~min}$ in a lunch room (INRA, STLO), then sealed with plastic paraffin film and incubated at $10^{\circ} \mathrm{C}$. Concerning semi-hard cheeses, 3 weeks ripened semi-hard cheeses of each condition were sliced $(60 \mathrm{~mm}$ x $50 \mathrm{~mm}$ x $8 \mathrm{~mm}$ ). The obtained slices were then exposed for $20 \mathrm{~min}$ in ten $60-\mathrm{mm}$ Petri dishes. Plates were then sealed and incubated at $12^{\circ} \mathrm{C}$. In both cases, fungal growth at the surface was visually evaluated after 10 days. Fungal contaminants were isolated on M2Lev agar $(20 \mathrm{~g} / \mathrm{L}$ malt extract, $3 \mathrm{~g} / \mathrm{L}$ yeast extract and $15 \mathrm{~g} / \mathrm{L}$ agar, $5 \mathrm{mg} / \mathrm{l}$ penicillin, $5 \mathrm{mg} / \mathrm{l}$ streptomycin). Isolates were then characterized at the genus level using phenotypic methods including macro- and microscopic observations (Pitt and Hocking, 2009) and for species identification, specific taxonomical markers were targeted. DNA regions of isolated yeasts and molds were amplified, sequenced and identified as detailed by Garnier et al. (2016).

\section{Sensory Analyses}

The impact of antifungal cultures on the sensorial properties of the produced sour creams and semi-hard cheeses was evaluated using a sorting task by a panel of 27-30 untrained judges. Sour cream samples were stored at $4^{\circ} \mathrm{C}$ for 15 days before sensory evaluation and $10 \mathrm{~g}$ of each sample was presented in disposable cups. Semi-hard cheeses were evaluated after 4 weeks ripening. Cheese rind was first brushed and wiped to harmonize the aspect of the samples and presented as $15 \mathrm{~g}(50 \times 40 \times 8 \mathrm{~mm})$ slices. Samples were left for $\sim 30 \mathrm{~min}$ at room temperature before being served, coded using a random 3-digit code and presented in a different, counterbalanced order for each judge. Control sour cream and cheese (with no antifungal culture) were presented in duplicate, leading to a total of 10 and 5 samples per session, respectively, for cream and cheese. Panelists were instructed to group together the samples perceived as the most similar, taking into account the characteristics they considered as important to differentiate the products. Once groups were made, panelists had to associate specific descriptors (Supplementary Table 1) to each group. Data were analyzed as recommended by Lê and Worch (2014) in the $R$ free software using the FactorMineR package by generating a contingency table (descfreq function) that calculated the number of occurrences of each descriptor in the different samples $(p<0.2)$. Then, a correspondence analysis (CA) was performed from the contingency table and plotted with confidence ellipse (functions plot.CA and ellipseCA).

\section{RESULTS}

\section{Antifungal Activity Screening}

Among the 32 screened strains (Table 1), lactobacilli showed higher antifungal activities than leuconostocs and propionibacteria. All strains except Propionibacterium acidipropionici CIRM-BIA928 showed antifungal activity against at least 1 fungal target in model cheese or yogurt. The antifungal activity varied between strains for all tested species. P. commune was the most inhibited fungal target followed by $M$. racemosus, while the G. geotrichum and Y. lipolytica yeasts were the most resistant as they were only inhibited by 1 Leuconostoc and 6 lactobacilli strains, with a very low $(+)$ or slight $(++)$ antifungal activity.

Antifungal strains showed a specific antifungal activity in each dairy model. The global score, i.e., the mean score of the 4 fungal targets tested, showed that 25 strains exhibited a higher or similar antifungal activity in cheese compared to yogurt, while it was the reverse for 6 strains ( $5 \mathrm{~L}$. rhamnosus and $1 \mathrm{~L}$. plantarum). P. acidipropionici CIRM-BIA928, Propionibacterium jensenii CIRM-BIA1774, and P. thoeni CIRM-BIA1763 were the only propionibacteria strain that exhibited antifungal activity in both models. In yogurt, 6 Propionibacterium and 1 Leuconostoc strains showed no antifungal activity. Antifungal activity for all strains was markedly higher when inoculated at $10^{7}$ compared to $10^{5}$ $\mathrm{CFU} / \mathrm{mL}$. At $10^{5} \mathrm{CFU} / \mathrm{mL}$, all strains exhibited very low (+) or no inhibition in yogurt while 7 lactobacilli, 1 leuconostoc strains and the reference cultures $\mathrm{X} 1$ and $\mathrm{X} 2$ showed a slight $(++)$ to total $(+++)$ inhibition in model cheese.

From the first screening (S1), 15 LAB strains (12 lactobacilli and 3 leuconostocs noted with a ${ }^{*}$ in Table 1) showed a high global score from 1.25 to 2.75 at least in one model. Among propionibacteria, the highest global score 0.75 was observed for 3 strains including P. acidipropionici CIRM-BIA1664 and CIRMBIA1763, and P. jensenii CIRM-BIA1785. Among the strains 


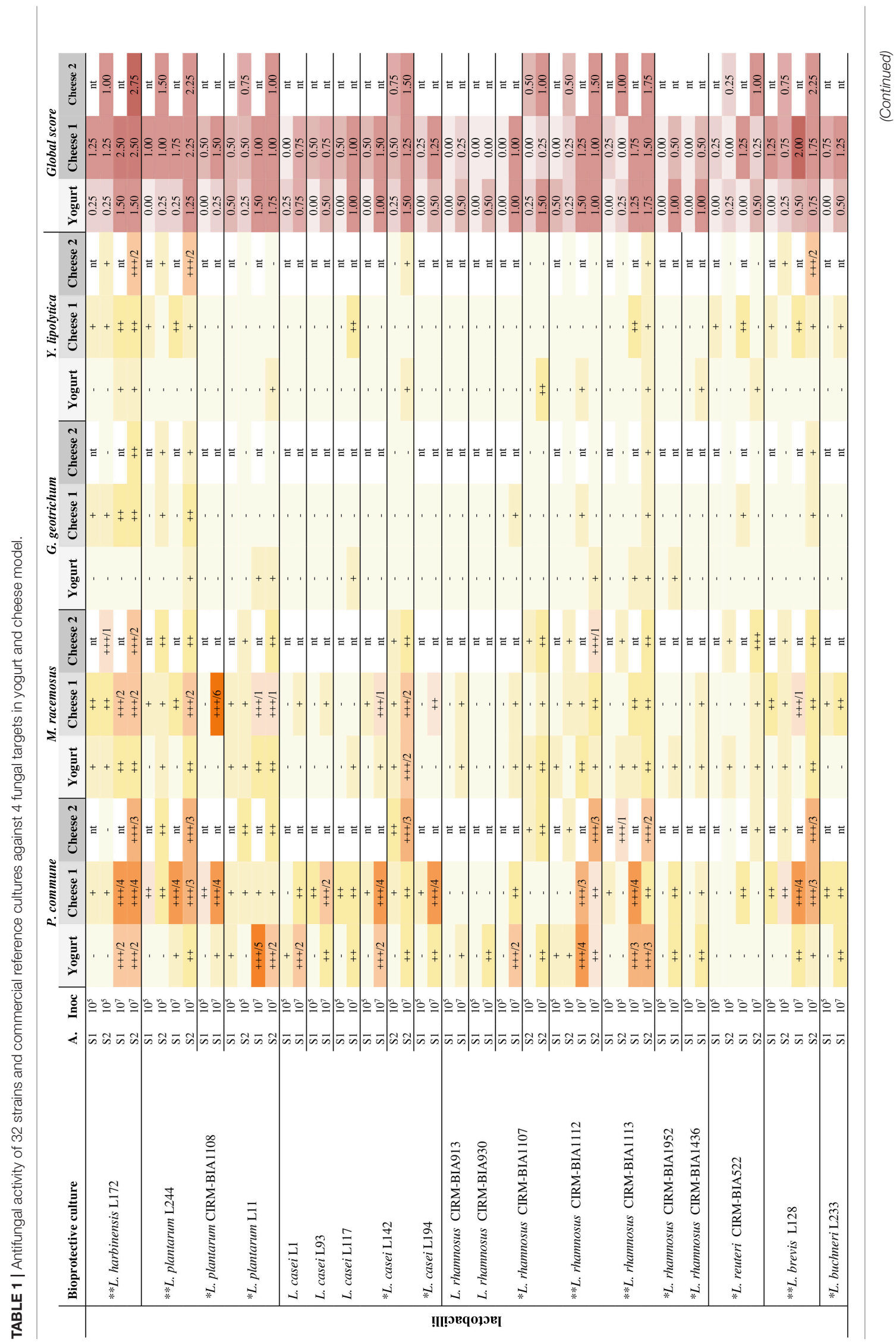




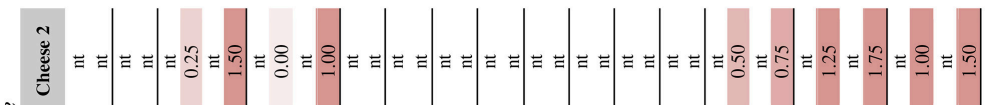

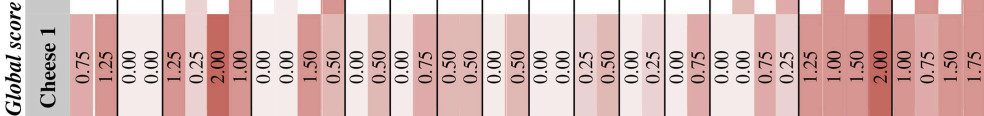

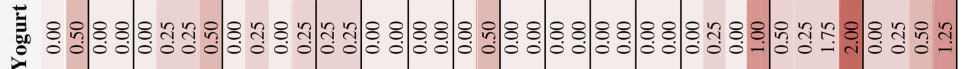

ปัँ

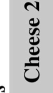

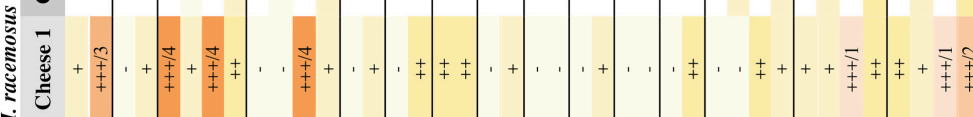

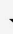

言

言

言

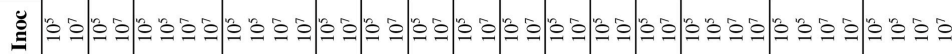

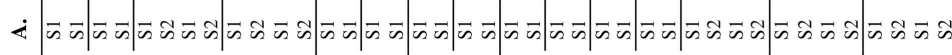

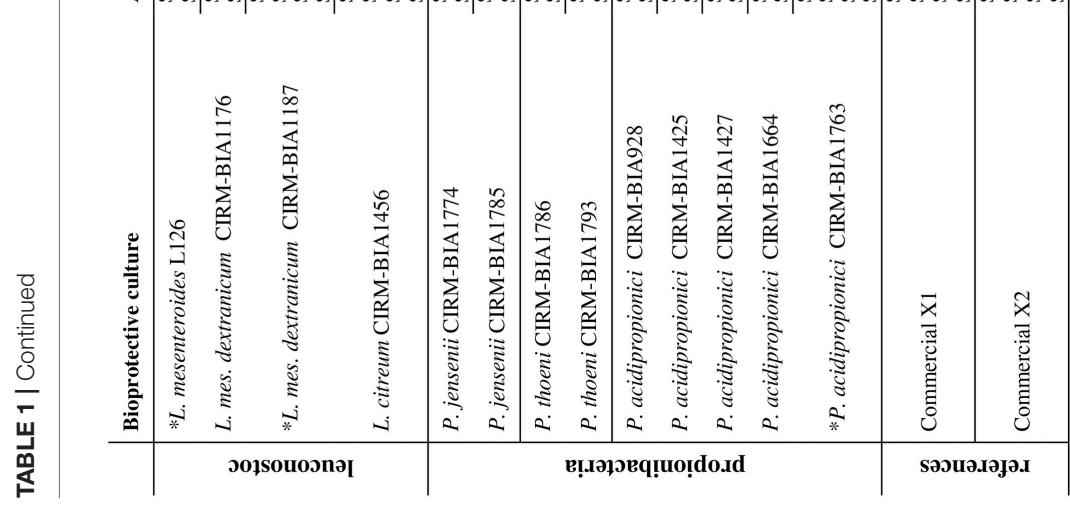

:

巡

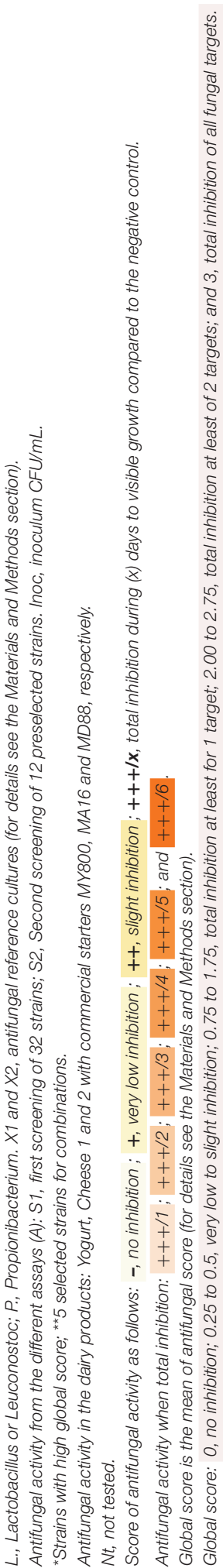


exhibiting the highest antifungal activity, 12 were selected for the second screening. Reference cultures X1 and X2 showed high global scores ranging from 1.25 to 2 , and $\mathrm{X} 1$ inhibited $P$. commune and $M$. racemosus 3-4 days more than $\mathrm{X} 2$.

The second screening (S2) showed little variations of the antifungal activity with the different commercial starters, indicating that starters did not impact the antifungal activity of the 12 strains (Table 1).

Based on the screening data (Table 1), 5 lactobacilli strains (noted with ${ }^{* *}$ in Table 1) were selected based on their antifungal activity equal or higher than that of the reference cultures and/or their activity spectrum in at least one dairy model. The selected strains were Lactobacillus harbinensis L172, which showed the highest global scores in both models, L. brevis L128 and L. plantarum L244, which showed the highest global scores in model cheese, and L. plantarum CIRM-BIA1113 and CIRMBIA1112, which showed the broadest activity spectra.

\section{Antifungal Activity of Strain Combinations Combination Test}

Based on the 5 selected strains, the 10 possible binary and 3 ternary strain combinations were evaluated in the yogurt and cheese models. Ternary combinations were configured to test the effect of combining the high antifungal activity of $L$. harbinensis L172, L. brevis L128, and L. plantarum L244 with 1 of the 2 L. rhamnosus strains that showed antifungal activity against the resistant G. geotrichum and Y. lipolytica fungi. However, these ternary combinations did not show any antifungal activity improvement compared to the corresponding pure cultures and binary associations (data not shown). Four binary combinations, A1, A2, A3, and A4, showed the highest antifungal activity among the 13 tested combinations (data not shown). The four binary combinations involved 4 strains, in particular L. plantarum L244 used in 3 combinations, and L. harbinensis L172 and L. brevis L128, used in 2 combinations.

In yogurt, A1 and A2 combinations showed the highest inhibition time (4 days) on $P$. commune growth in the third screening. The inhibition obtained with the A3 and A4 combinations, 2 or 3 days for P. commune, was similar to pure cultures having the highest antifungal activity (i.e., L. plantarum L244 and L. harbinensis L172). Combination A4 showed the highest antifungal activity against G. geotrichum in yogurt with an inhibition for 1 day vs. no or low inhibition and no or slight inhibition for the other combinations.

In cheese model, combinations A1, A2, and A3 showed a broad spectrum, with a high inhibition of 1-4 days of $P$. commune and $M$. racemosus, and a slight to total inhibition of G. geotrichum and $Y$. lipolytica. Regarding inhibition of $P$. commune, the number of inhibition days induced by A1, A2, A3, and A4 were similar to that of the strains that showed the highest inhibition in pure cultures. A1 and A2 combinations showed inhibition of 1 day of G. geotrichum, while A2 was the only combination inhibiting $Y$. lipolytica growth for the same duration.

Before pilot scale applications, a safety evaluation of the strains composing the different combinations was performed. In this context, biogenic amine production and antibiotics resistance were studied. Combinations A2 and A4 were excluded because of the risk of biogenic amine production by L. brevis L128, which possesses both the $\operatorname{tyrDC}$ and $a g D i$ genes, as recently demonstrated (Coton et al., 2018). These genes, encoding for a tyrosine decarboxylase and an agmatine deiminase, are associated with potential production of tyramine and putrescine, respectively. Therefore, only the A1 and A3 combinations were then kept for following experiments.

\section{Determination of the Optimal Inoculum for Antifungal Activity and Action Spectrum}

The minimal inoculum allowing high antifungal activity was assessed using 3 intermediate levels of inoculation for combinations A1 and A3, selected from the previously obtained results.

In yogurt, the highest antifungal activity was always observed when strains were inoculated at the highest level, i.e., $2.10^{7}$ $\mathrm{CFU} / \mathrm{mL}$ (Table 2). In contrast, in cheese, inoculum could be lower depending on the target $\left(10^{7} \mathrm{CFU} / \mathrm{mL}\right.$ and even $2.10^{6}$ $\mathrm{CFU} / \mathrm{mL}$ in some cases). Combinations A1 and A3 showed little variations of antifungal activity with the different tested commercial starters (data not shown).

As for the antifungal activity spectrum, the six additional fungal targets ( $P$. bialowiezense, $P$. pinodella, $R$. mucilaginosa, M. guilliermondii, C. parapsilosis, and T. asahii) were more sensitive than the initial 4 fungal targets. In particular, the time to visible growth reached at least 1 day and more than 15 days in many cases (Table 2). It should be noted that T. asahii did not grow in yogurt therefore the inhibition against this target could not be established in this model. Combination A1 showed the widest spectrum and led to the highest inhibition time in both models against the six additional fungal targets with a global score of 3 in yogurt and cheese, and was associated with 2-15 days of inhibition (Table 2). At the two levels of inoculum $\left(10^{7}\right.$ and $2.10^{7} \mathrm{CFU} / \mathrm{mL}$ ) in yogurt, the A3 combination totally inhibited at least 6 of the 9 tested fungi, for a duration ranging from 2 to 8 days. The A3 combinations showed a similar spectrum than that of A1. Nevertheless, A1 did not inhibit R. mucilaginosa (yogurt), and only and slightly inhibited M. guilliermondii and R. mucilaginosa (cheese). Combination A3 showed a slight inhibition of $R$. mucilaginosa and C. parapsilosis in yogurt.

The activity spectrum of A1 and A3 was compared to the corresponding pure cultures, against 10 fungal targets in cheese model and 9 in yogurt, at $10^{7}$ and $2.10^{7} \mathrm{UFC} / \mathrm{mL}$ (Line S6 from Table 2). In half of the cases, the time to visible growth increased by 1-11 days for the combinations compared to the corresponding pure cultures. In $64 \%$ of cases, it did not differ from the strain showing the highest activity in pure culture, and in $4 \%$ of cases, it decreased.

\section{In Situ Application of Antifungal Combinations in Sour Cream and Semi-hard Cheese}

These pilot scale applications consisted in evaluating the ability of two selected combinations, A1 and A3, to inhibit fungi in challenge and shelf life tests in sour cream and semi-hard cheese. 


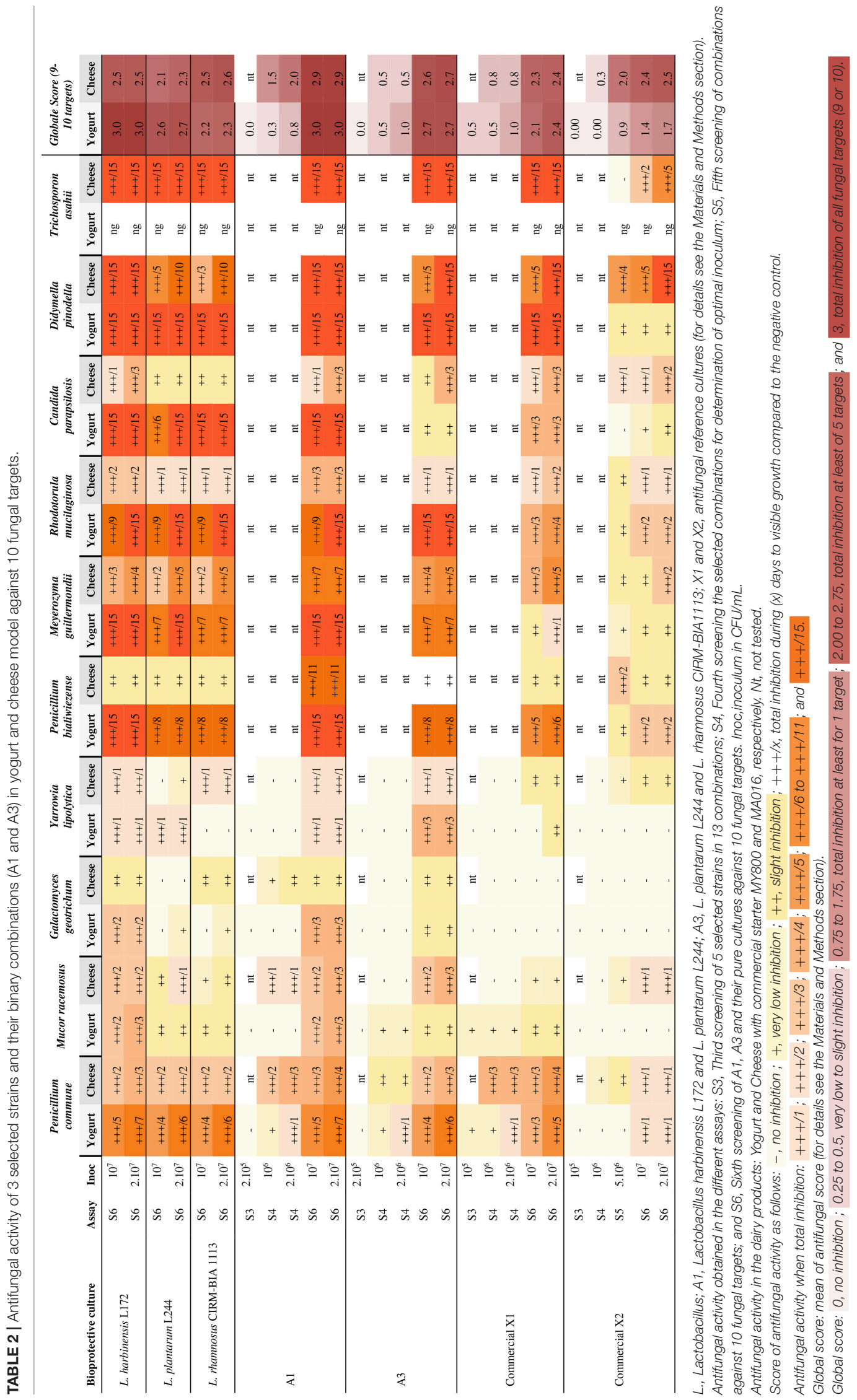



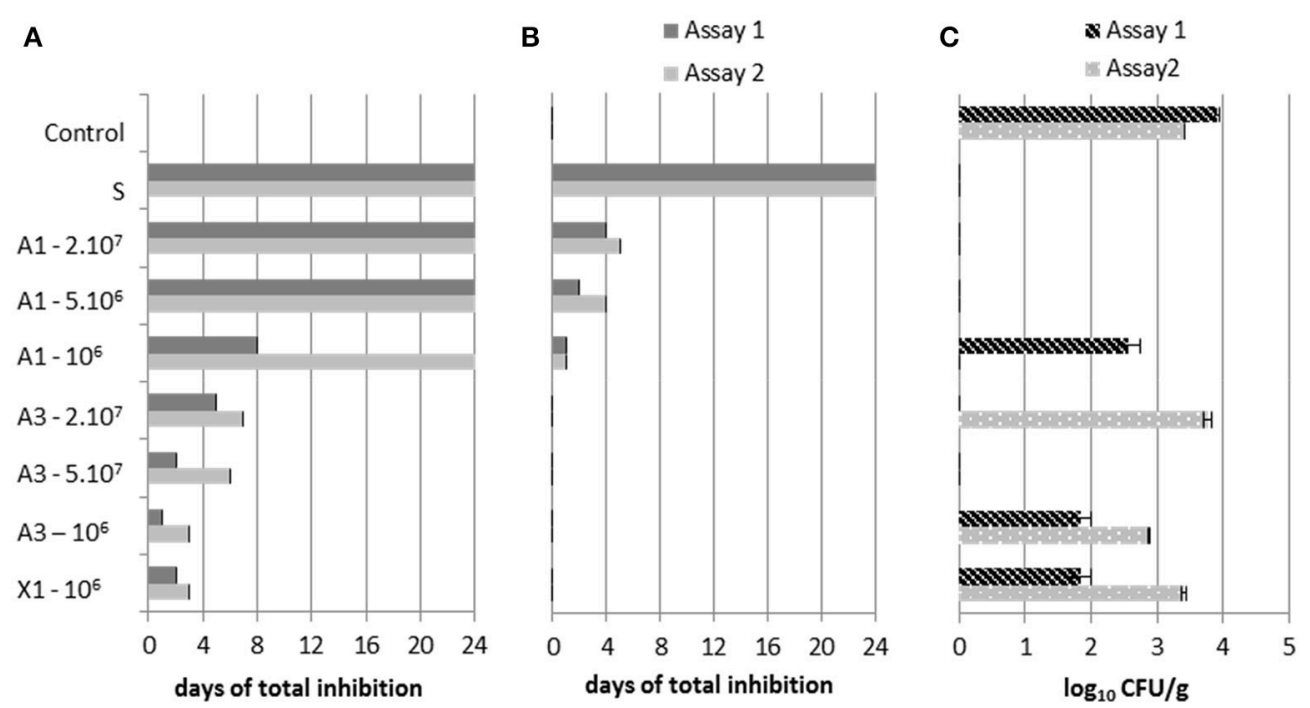

FIGURE 2 | Effect of combinations A1, A3 and X1 (for details see the Materials and Methods section) at different inoculation levels $2.10^{7}, 5.10^{6}$, and $10^{6} \mathrm{CFU} \mathrm{mL}$, sorbate (S, positive control) and control (negative control, no antifungal agent added) in sour cream against $P$. commune (A) and $M$. racemosus (B) (antifungal activity was expressed in days to visible growth compared to the negative control); and $R$. mucilaginosa (C) (yeast counts after storage for 7 days at $10^{\circ} \mathrm{C}$, expressed as $\left.\log _{10} \mathrm{CFU} / \mathrm{g}\right)$.

\section{Challenge-Tests}

Inoculation of the fungal targets was performed to simulate a post-processing contamination. Potassium sorbate and natamycin were used as chemical additive reference (positive controls) for sour cream and semi-hard cheese, respectively.

In sour cream, the fungal target $P$. commune was the most inhibited, followed by $R$. mucilaginosa and M. racemosus, which were inhibited during a maximal time of 7 days compared to 24 days for $P$. commune (Figure 2). The A1 combination showed higher inhibition of the 3 targets compared to A3 and the reference culture $\mathrm{X} 1$, and an equal inhibition to that of sorbate (24 days) against $P$. commune, although some variability was observed. A1 antifungal activity led to 1 to 24 days inhibition of $M$. racemosus and $P$. commune (Figures $\mathbf{2 A}, \mathbf{B}$ ). In sour cream containing $\mathrm{A} 1$ at the 3 inoculum levels, $R$. mucilaginosa was not detectable $(<1$ cell/g) after 7 days (Figure $2 \mathrm{C}$ ). At the 3 tested concentrations, A3 slightly inhibited (++) M. racemosus (data not shown) and totally inhibited $P$. commune for 1-7 days (Figure 2A). In one of sour cream assays, A3 only inhibited R. mucilaginosa at a $5 \cdot 10^{6} \mathrm{CFU} / \mathrm{mL}$ inoculum level (Figure 2C). $\mathrm{X} 1$ inoculated at $10^{6} \mathrm{CFU} / \mathrm{mL}$ showed a similar antifungal activity to that of A3 inoculated at the same concentration. Some variability in antifungal activity was observed between replicates. This was especially true for inhibition of $P$. commune and $R$. mucilaginosa by the A1 combination at the lowest inoculation level.

In semi-hard cheeses, in the second assay, M. racemosus was totally inhibited for 1 day by A3 (Figure 3) and slightly inhibited $(++)$ by A1 (data not shown). The fungal target $P$. commune was more inhibited by A1 and the commercial culture CC 3,1 to 6 days depending on the assay (Figure 3A). Antifungal activity of bioprotective cultures and natamycin varied between assays.

\section{Shelf Life Tests}

Shelf life tests were performed on sour cream and semi-hard cheese to evaluate the growth of naturally contaminating fungi and to get closer to actual food post-contamination in particular in terms of inoculum $(\sim 1$ spore in natural contamination vs. 50 spores as used as a worst case scenario in the previous experiments). For negative control sour creams (without potassium sorbate or antifungal culture), $100 \%$ of samples were contaminated. In contrast, for all the other treatments, no contamination of cream samples was observed with exception of the sour cream inoculated with $\mathrm{A} 3$ at $5.10^{6} \mathrm{CFU} / \mathrm{mL}$, which presented $10 \%$ of contaminated samples (Figure 4). The natural fungal contaminants found on contaminated sour cream with A3 were identified as Cladosporium allicinum and Exophiala xenobiotica, while the fungal contaminants found in control sour cream included the 2 later mentioned fungi and 6 other species, namely Penicillium crustosum, Penicillium glabrum, Cladosporium cladosporioides, D. hansenii, Bulleromyces albus, and Sporidiobolus metaroseus. In semi-hard cheese, it is worth noting that after 4 weeks in non-sterile ripening chambers at $12^{\circ} \mathrm{C}$, the cheese surface was colonized in all assays with molds, therefore fungal growth was evaluated on the slice surface after 10 days of incubation post-slicing. The totality of negative control cheeses samples were contaminated, whereas samples of A1, A3 and commercial culture CC3 supplemented cheeses were notably less contaminated as shown on Figure 5. The fungal contaminant on cheese slices was identified as Penicillium crustosum.

\section{Microbial Enumeration and $\mathrm{pH}$}

Antifungal populations were evaluated by enumeration of the total lactobacilli in sour cream and semi-hard cheese in order to verify their viability at inoculation time, their growth and 


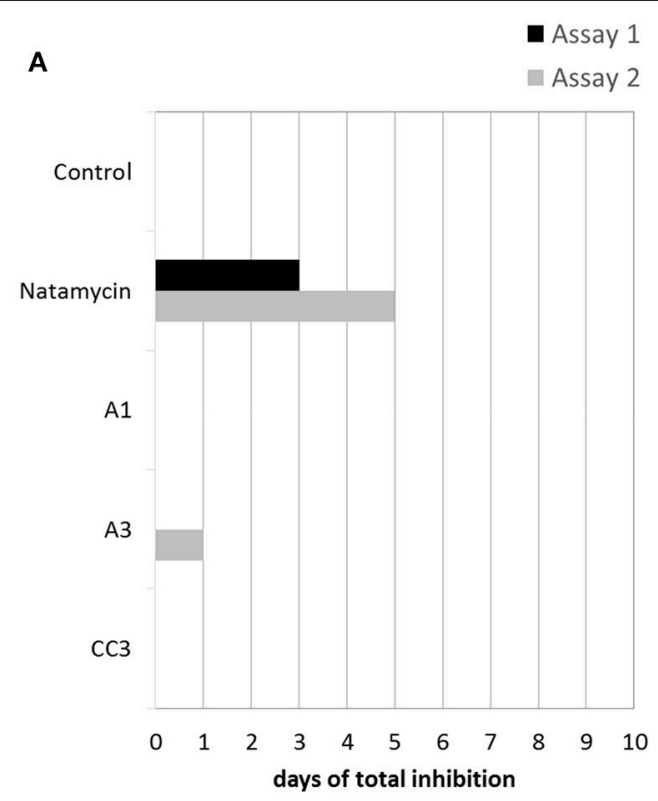

B

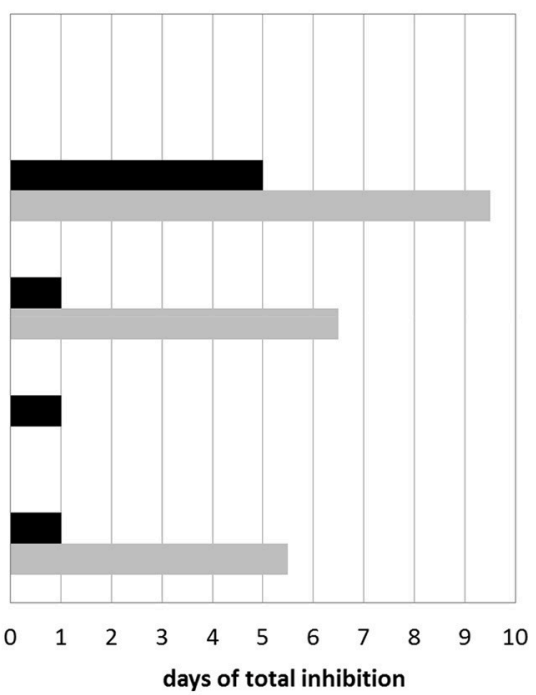

FIGURE 3 | Antifungal activity of natamycin, and the combinations A1, A3 and commercial antifungal culture CC3 inoculated at 5.106 CFU/mL on semi-hard cheese against $P$. commune (A) and $M$. racemosus (B) (antifungal activity was expressed in days to visible growth compared to the negative control).

survival at different stages of manufacture and storage. Samples containing A1 and A3 presented two types of lactobacilli colonies with similar number on the plates, corresponding to the two inoculated strains constituting the antifungal combinations.

The determined populations of antifungal cultures in cream and cheese milk were in agreement with the targeted inoculum concentrations and showed similar changes during the process regardless of the considered antifungal culture. A1 and A3 combination populations were stable or even increased during cheese ripening with maximal values of $\sim 8.5 \log _{10} \mathrm{CFU} / \mathrm{g}$ (Figure 6).

There was no significant difference of $\mathrm{pH}$ during acidification between sour cream with and without antifungal cultures. Nevertheless, after 4 weeks of storage at $10^{\circ} \mathrm{C}$, the $\mathrm{pH}$ of cream inoculated at $2.10^{7} \mathrm{CFU} / \mathrm{mL}$ with both $\mathrm{A} 1$ and $\mathrm{A} 3$ combinations was lower (0.2 pH units less) than that of control creams. In semihard cheese, antifungal cultures did not impact $\mathrm{pH}$ neither during manufacture nor during ripening.

Starter culture populations in sour cream and semi-hard cheeses with antifungal cultures did not differ, during the fermentation and 4 weeks of storage/ripening, from those of the control samples (data not shown). No NSLAB (Non Starter Lactic Acid Bacteria) was detected on LAMVAB on the control products. Bacterial population slightly varied during storage/ripening or sour cream and semi-hard cheese. In all products, starter culture population decreased in both products from the second weeks of storage. Concerning antifungal cultures, A1, A3 and X1 population increased during the first week of storage in sour cream. In semi-hard cheese, A1 and A3 population slightly increased during the 4 ripening weeks (data not shown).

\section{Sensory Analyses}

The CA map built from sensory evaluation data of sour cream showed that the samples were mainly separated on dimension 1 and cream with sorbate was separated from all other samples on dimension 2 (Figure 7A). The negative side of dimension 1 in the map contained 6 out of the 7 creams with antifungal cultures including the reference culture $\mathrm{X} 1$, characterized by their more acidic flavor (Table 3). Only the cream with A1 at $10^{6} \mathrm{CFU} / \mathrm{mL}$ was perceived similar to the control samples and characterized by a "mild flavor."

For cheeses, the CA map separates control cheeses from the other cheese samples on dimension 2 and the cheese containing the A1 culture on dimension 2. Control cheeses were characterized as tasteless, whereas cheese with CC3 was described as slightly acidic and with a different texture, and cheese A1 associated with a more bitter and acidic flavor (Figure $7 \mathbf{B}$, Table 4).

\section{DISCUSSION}

In the first part of this work, the antifungal activity of 32 strains for potential use as adjunct antifungal cultures was evaluated using a high-throughput screening method in two dairy models against 4 fungal targets, namely $P$. commune, $M$. racemosus, $G$. geotrichum, and Y. lipolytica. As shown in several studies, the choice of the screening media is a crucial step because the number of potential antifungal agents can be drastically reduced between a screening step performed in synthetic media and in situ tests in actual food products. For example, Delavenne et al. (2013) indicated that from 11 antifungal LAB active in vitro, only 1 showed a very strong antifungal activity in yogurt. Similarly, Le 


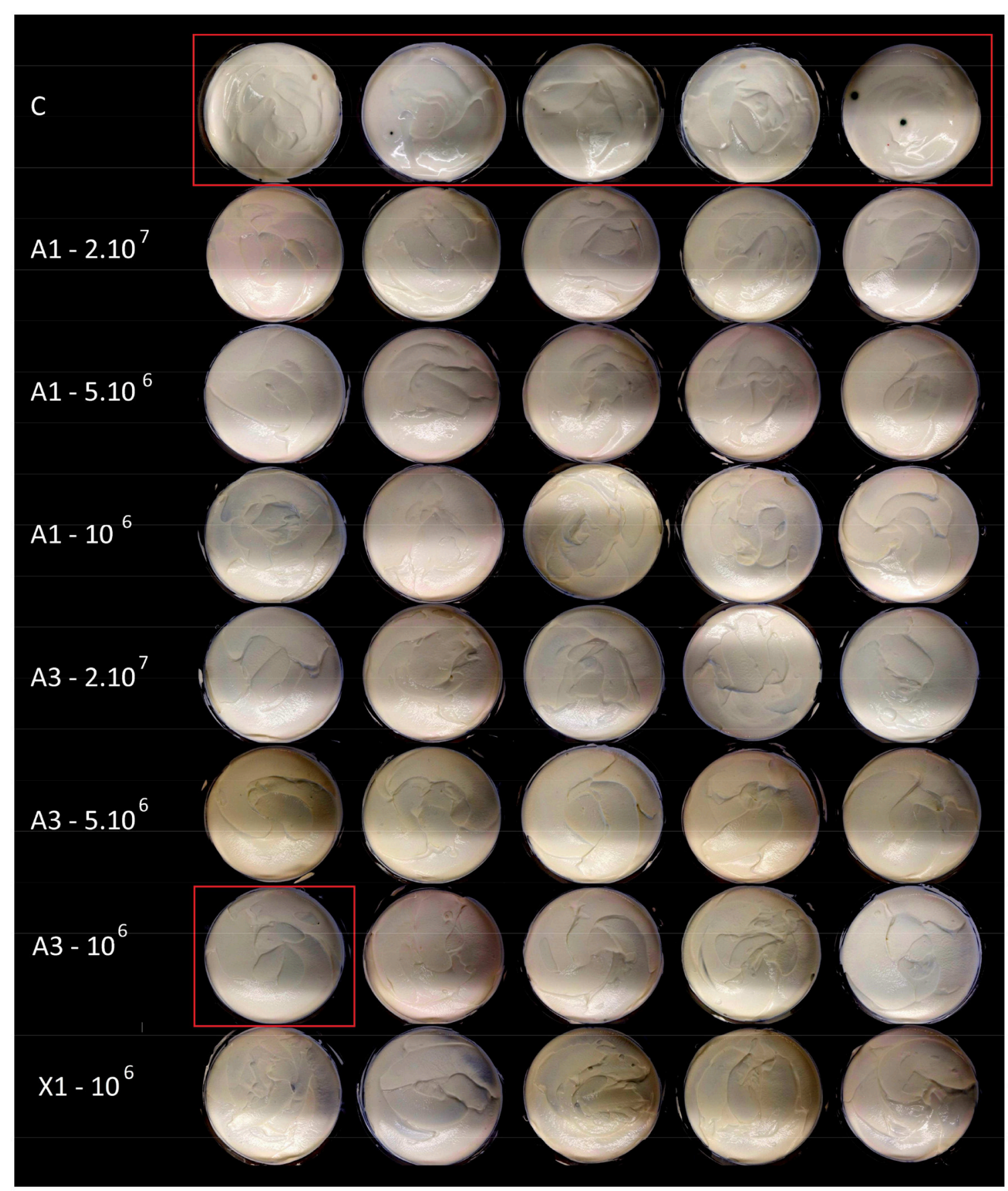

FIGURE 4 | Photographs showing sour cream surfaces exposed to natural contamination by environmental fungi followed by incubation for 10 days at $10^{\circ} \mathrm{C}$. Cream with no antifungal agent (C), and antifungal cultures $\mathrm{A} 1, \mathrm{~A} 3$, and $\mathrm{X} 1$ ( $\mathrm{X} 1=$ reference culture, for details see the Materials and Methods section). Inoculation levels are expressed in $\mathrm{CFU} / \mathrm{mL}$ and contaminated samples are highlighted in red.

Lay et al. (2016b) showed that the number of LAB active in bakery products was significantly lower than that observed on MRS and WFH media. In order to avoid this bottleneck, in this study, two media closely related to actual dairy products were chosen, a cheese-mimicking model previously developed by Garnier et al. (2018) and yogurt adapted to high-throughput screening in this study. They were both relevant to the final purpose of developing antifungal cultures compatible with various dairy technologies. The use of this screening method allowed preparing more than 5,000 miniaturized yogurts and model cheeses required to test the antifungal candidates, in the presence of different commercial starters, against up to 10 fungal targets. A difference 


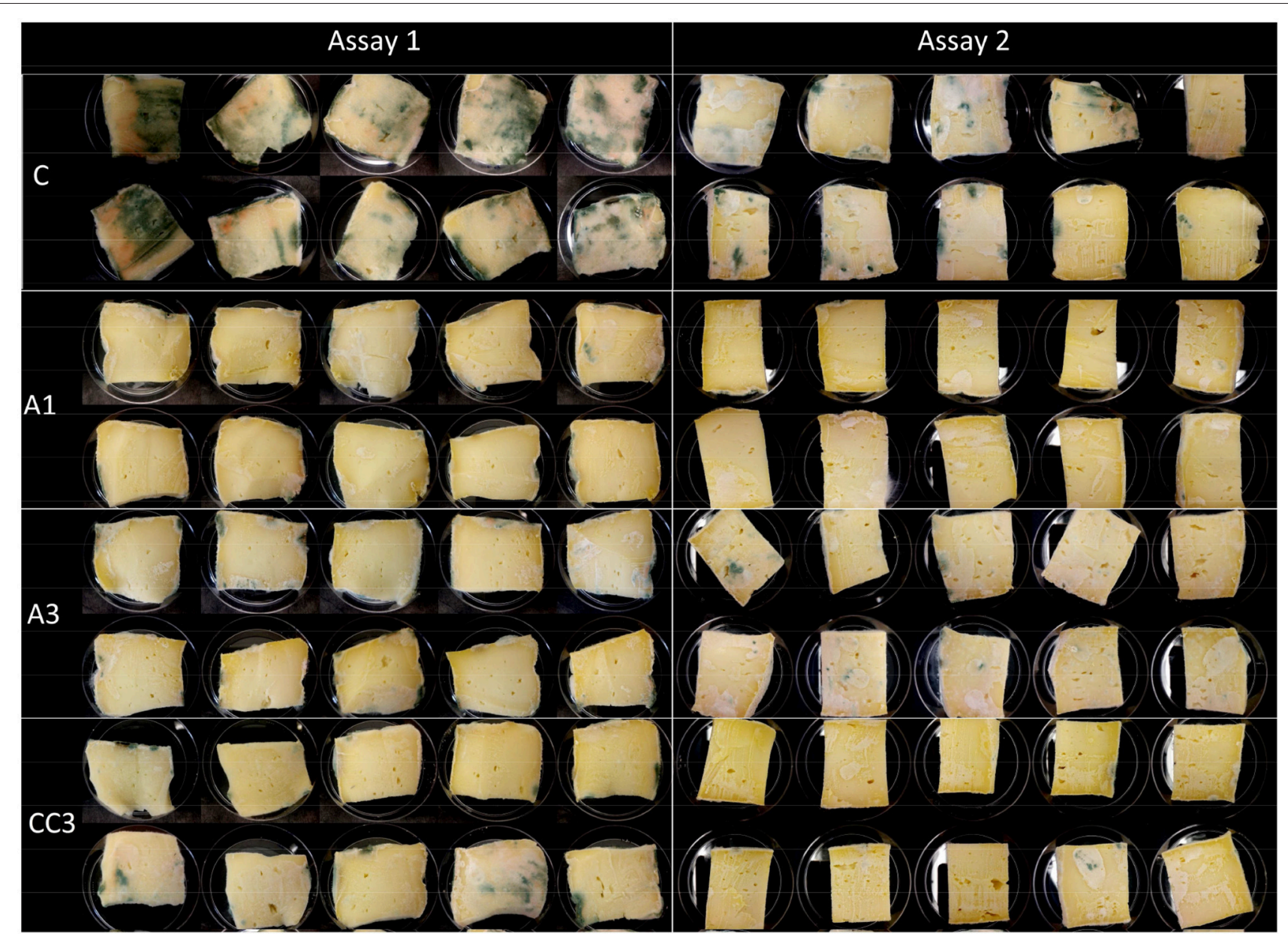

FIGURE 5 | Photographs showing semi-hard cheese slices exposed to natural contamination by environmental fungi followed by incubation for 10 days at $10^{\circ} \mathrm{C}$. Cheeses with no antifungal agent (Control) and antifungal cultures A1, A3, and CC3 (commercial antifungal culture) at 5.106 CFU/mL.

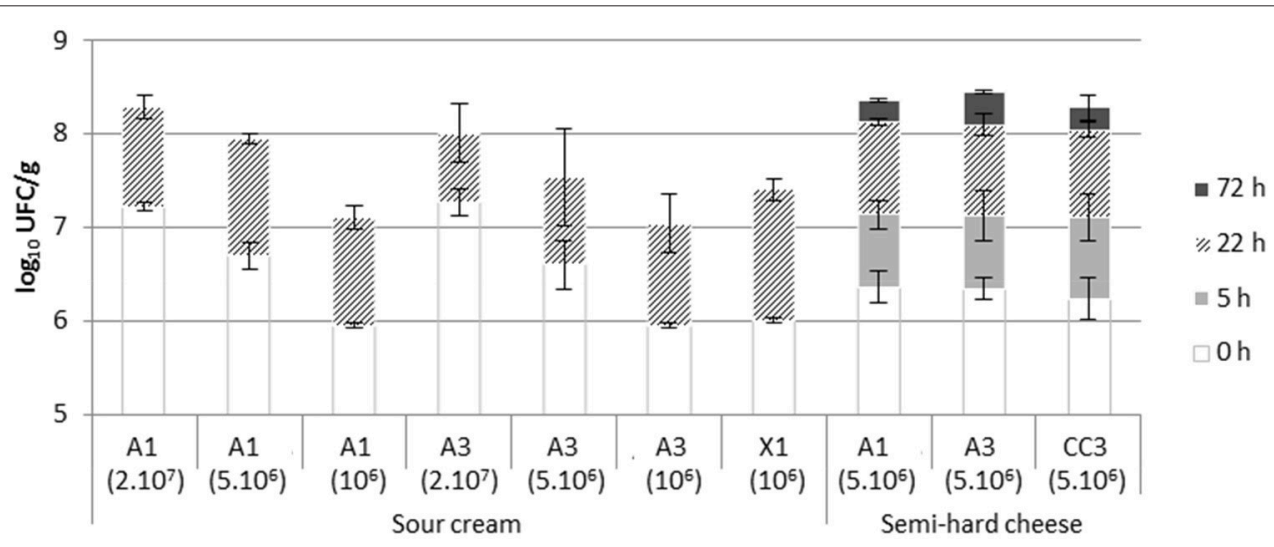

FIGURE 6 | Populations of antifungal bacteria (A1, A3, X1, or CC3, see Materials and Methods section for details) inoculated at $2.10^{7}$, $5.10^{6}$, and $10^{6}$ CFU/mL in sour cream and semi-hard cheese at inoculation time $(\mathrm{O} h)$ and at the end of fermentation (22 h) and, for cheese only, after curd press ( $5 \mathrm{~h})$ and smear treatment (72 h).

of antifungal activity was observed between the two dairy models, thus demonstrating the importance of matrix (composition) and production condition (time of incubation, temperature).
No significant impact of the 5 commercial starters tested in yogurt and cheese models was observed on the antifungal activity. This suggested low interaction (metabiosis) between the starter 

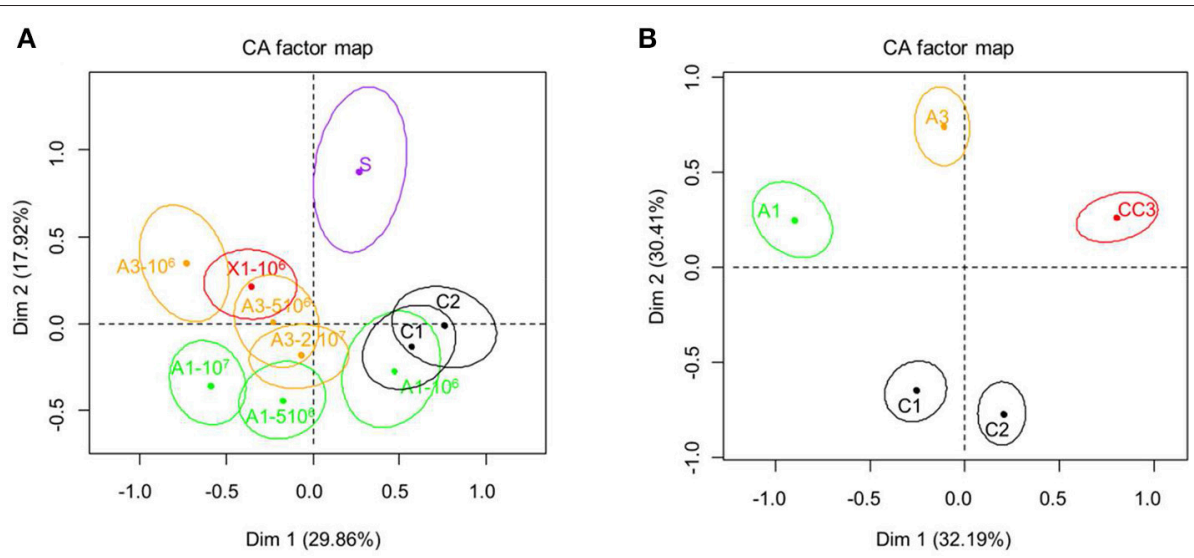

FIGURE 7 | Correspondence analysis map of sensory analysis data obtained for the sour cream (A) and semi-hard cheeses (B) samples. For sour cream samples: no antifungal agent (negative controls = C1 and C2), potassium sorbate at 0.08\% (S) (positive control) and antifungal cultures A1, A3, and X1 (see Materials and Methods section for details) at $2.10^{7}, 5.10^{6}$, and $10^{6} \mathrm{CFU} / \mathrm{mL}$. For semi-hard cheeses: no antifungal agent (negative controls = C1 and C2), antifungal bioprotective cultures $\mathrm{A} 1, \mathrm{~A} 3$, and CC3 (commercial antifungal culture) at $5.10^{6} \mathrm{CFU} / \mathrm{mL}$.

cultures and the antifungal adjunct cultures. The choice of fungal targets is also crucial as they should be representative of contaminants in terms of occurrence and biodiversity. In this study, fungal target strains were selected from contaminated dairy products (Garnier et al., 2016) among species known as frequent contaminants in dairy products (Pitt and Hocking, 2009). Noteworthy, in the present study, the 4 fungal targets used for the screening steps were more resistant, in particular G. geotrichum and Y. lipolytica, than the 6 additional fungi used during the activity spectrum evaluation. In agreement with our observations, the two latter species were reported to be more resistant to chemical preservatives than other fungal species, e.g., for G. geotrichum and Y. lipolytica strains, the minimal inhibitory concentration of potassium sorbate at $\mathrm{pH} 5$ in PDA was $1 \mathrm{~g} / \mathrm{L}$ in contrast to $0.3 \mathrm{~g} / \mathrm{L}$ for P. commune (Garnier et al., 2016). Similarly, the antifungal activity of LAB strains against cereal and bakery fungal contaminants was reported to differ for all the tested fungal targets (Inglin et al., 2015; Le Lay et al., 2016b; Russo et al., 2017).

The tested Lactobacillus strains globally showed the highest antifungal activity in one or both of the dairy models, compared to Leuconostoc and Propionibacterium strains, even if the activity was strain-dependent within the species, as expected. Many Lactobacillus species have been previously reported to exhibit antifungal activity in dairy products. For example, in yogurt, several authors showed that various strains belonging to the L. harbinensis, L. rhamnosus, L. paracasei or L. casei species were active against Penicillium spp., but also, for some of them, against D. hansenii, K. lactis, Kluyveromyces marxianus, R. mucilaginosa, and Y. lipolytica (Delavenne et al., 2013, 2015; Li et al., 2013; Aunsbjerg et al., 2015). In cottage and cheddar cheese, different studies showed that $L$. reuteri, L. amylovorus, L. plantarum and L. rhamnosus strains showed antifungal activity against Penicillium spp. (Cheong et al., 2014; Lynch et al., 2014; Fernandez et al., 2017). Assessment of the antifungal activity of 13 $\mathrm{LAB}$ strains toward 3 ochratoxin A-producing fungi in synthetic media showed that L. plantarum B4496, L. brevis 207 and 3 other LAB strains inhibited the tested fungal targets (Ngang et al., 2015). The lower antifungal activities observed for Leuconostoc and Propionibacterium strains, despite their reported capacity to produce antifungal organic acids, could result from their inability to reach high populations under the screening conditions (i.e., incubation for $4-6 \mathrm{~h}$ at $42^{\circ} \mathrm{C}$ in yogurt and for $72 \mathrm{~h}$ at $20^{\circ} \mathrm{C}$ in cheese). Leuconostoc spp. optimally grow at temperatures ranging between 20 and $30^{\circ} \mathrm{C}$ (Martley and Crow, 1993; Hemme and Foucaud-Scheunemann, 2004). This aspect may explain the low antifungal activity of Leuconostoc strains observed in the present study in yogurt. Concerning Propionibacterium spp., their slow growth rate can explain their low activity observed. The generation time of $P$. freudenreichii is around 5-9h under optimal conditions, i.e., in a complex laboratory medium at $30^{\circ} \mathrm{C}$ (Thierry et al., 2002), and $15-30 \mathrm{~h}$ in cheese and cheese juice at $24^{\circ} \mathrm{C}$ (Salvat-Brunaud et al., 1997). Antibiosis, via the production of antifungal molecules, and $\mathrm{pH}$ decrease are the main factors contributing to $\mathrm{LAB}$ and $\mathrm{PAB}$ antifungal activity in foods (Leyva Salas et al., 2017). The production of antifungal compounds (e.g., lactic, acetic, succinic, propionic, formic, and butyric acids) by lactobacilli and pediococci strains was shown to vary significantly depending on the medium (Özcelik et al., 2016). There are only few studies concerning the antifungal activity of Leuconostoc compared to those concerning Lactobacillus spp. and most of them were performed in vitro. For example, two L. citreum strains were shown to inhibit fungal contaminants of bakery products (Valerio et al., 2009; Baek et al., 2012; Le Lay et al., 2016b).

The binary combinations A1 and A3 showed the same broad spectrum as their constituting strains grown in pure cultures. They increased the time to visible growth of fungal targets in one third of cases (inhibition tested for 2 combinations, at 2 inoculations levels against 10 fungal targets in two dairy models). Some previous studies performed in different media also reported an improvement of the antifungal activity of binary combinations of strains, compared to pure cultures. This was 
TABLE 3 | Descriptors cited at a significantly $(p<0.2)$ higher frequency between sour creams (assay 1).

\begin{tabular}{|c|c|c|c|c|}
\hline Sample & Inoc. & Descriptors & Intern \% & $p$-value \\
\hline \multirow[t]{3}{*}{ Control 1} & - & Tasteless & 7.8 & 0.04 \\
\hline & & Odorless & 6.3 & 0.06 \\
\hline & & Mild flavor & 12.5 & 0.11 \\
\hline Control 2 & - & Mild flavor & 13.4 & 0.05 \\
\hline \multirow[t]{5}{*}{$\mathrm{A} 1$} & $2.10^{7}$ & Acidic & 23.4 & 0.03 \\
\hline & & After-taste & 3.1 & 0.16 \\
\hline & $5.10^{6}$ & Lactic & 6.2 & 0.14 \\
\hline & $10^{6}$ & Lactic & 15.2 & 0.02 \\
\hline & & Nutty after-taste & 3.0 & 0.17 \\
\hline \multirow[t]{6}{*}{ A3 } & $2.10^{7}$ & Slightly acidic & 6.0 & 0.18 \\
\hline & $5.10^{6}$ & Balanced & 3.2 & 0.10 \\
\hline & $10^{6}$ & Very acidic & 11.5 & 0.04 \\
\hline & & Pungent & 11.5 & 0.09 \\
\hline & & Acidic & 21.3 & 0.10 \\
\hline & & Pronounced flavor & 1.6 & 0.19 \\
\hline \multirow[t]{3}{*}{$X 1$} & $10^{6}$ & Mild flavor & 3.3 & 0.15 \\
\hline & & Sour cream & 1.6 & 0.19 \\
\hline & & Acidic & 19.7 & 0.20 \\
\hline \multirow[t]{4}{*}{ S } & - & Sweet & 3.1 & 0.02 \\
\hline & & Different after-taste & 3.1 & 0.10 \\
\hline & & Different taste & 3.1 & 0.16 \\
\hline & & Cheese flavor & 1.6 & 0.20 \\
\hline
\end{tabular}

Control 1 and 2, same sample, sour creams with no antifungal culture. A1, A3, and X1, sour cream inoculated with the antifungal cultures $A 1, A 3$, and $X 1$ ( $X 1=$ reference culture, for details see the Materials and Methods section), S, Sour cream with no antifungal culture and $0.08 \%$ of potassium sorbate, Inoc., Inoculum of the antifungal culture (CFU/mL), Intern \%, Percent of frequency for each descriptor inside the group. Descriptors were translated from french to english, for more details go to Supplementary Table 1.

the case for example for combinations of L. paracasei subsp. paracasei SM20 and $P$. jensenii SM11 in vitro (Schwenninger and Meile, 2004), P. thoenii P-127 and either L. rhamnosus B-445 or L. plantarum DSA 20174 in Kareish cheese (El-Shafei et al., 2008), and L. rhamnosus A238 and Bifidobacterium animalis subsp. lactis A026 in cottage cheese (Fernandez et al., 2017). The WO2012136830A1 patent applicable to yogurt reported that binary combinations ( $L$. paracasei CHCC12777 and L. rhamnosus CHCC12697 or L. paracasei CHCC12777 and L. rhamnosus CHCC14226) were more effective than either of the strains alone (Hornbaek et al., 2014). We hypothesized that the improvement of the antifungal activity of binary combinations consisting of L. plantarum L244 combined with either L. harbinensis L172 (A1), or L. rhamnosus CIRM-BIA1113 (A3) observed in this study was due to a higher quantity and diversity of the antifungal compounds produced compared to single strains, because it is known that antifungal compounds act in synergism and that Lactobacillus spp. produce various antifungal compounds (Crowley et al., 2013; Aunsbjerg et al., 2015; Le Lay et al., 2016a). Despite the fact that the A1 and A3 combinations most generally did not improve the antifungal activity to a great extent compared to the corresponding strains, we expect that using a combination of strains rather than a single
TABLE 4 | Descriptors cited at a significantly $(p<0.2)$ higher frequency between semi-hard cheese inoculated with antifungal combinations A1, A3 and commercial antifungal culture CC3 at $5.10^{6} \mathrm{CFU} / \mathrm{mL}$ and the non-inoculated control semi-hard cheeses (Control 1 and 2).

\begin{tabular}{|c|c|c|c|}
\hline Sample & Descriptors & Intern \% & $p$-value \\
\hline Control 1 & Tasteless & 14.05 & 0.07 \\
\hline \multirow[t]{2}{*}{ Control 2} & Tasteless & 16.36 & 0.01 \\
\hline & Not melting & 1.82 & 0.16 \\
\hline \multirow[t]{5}{*}{ A1 } & Some holes & 1.69 & 0.07 \\
\hline & Bitter taste & 1.69 & 0.07 \\
\hline & Pungent & 5.08 & 0.09 \\
\hline & Odorless & 2.54 & 0.10 \\
\hline & Acidic & 4.24 & 0.15 \\
\hline A3 & No significantly different descriptors & & \\
\hline \multirow[t]{5}{*}{ CC3 } & Smooth texture & 1.89 & 0.03 \\
\hline & Slightly acidic & 4.40 & 0.05 \\
\hline & No holes & 1.26 & 0.13 \\
\hline & Melting & 1.26 & 0.13 \\
\hline & Firm texture & 2.52 & 0.14 \\
\hline
\end{tabular}

Control 1 and 2, same semi-hard cheese sample with no antifungal culture. A1, A3, and CC3, semi-hard cheese inoculated with antifungal cultures A1, A3, and CC3 (CC3, commercial antifungal culture). Intern\%, Percent of frequency for each descriptor inside the group. Descriptors were translated from french to english, for more details go to Supplementary Table 1.

strain would increase the technological flexibility and the chance to see at least one of the strain expressing its antifungal activity in a diversity of conditions.

The in vitro screening and the challenge-tests performed in the present study are a worst case contamination scenario because 50 fungal spores were inoculated due to practical constraints, far above a natural contamination generally due to 1-2 contaminant spores. Moreover, cream was incubated at $10^{\circ} \mathrm{C}$ instead of $4^{\circ} \mathrm{C}$ as usually recommended for storage. Taken together, these aspects suggest that the antifungal activity could be even higher in real conditions, as strongly suggested by the shelf life test results. In sour cream, A1 and A3 combinations showed a bioprotective activity against natural fungal contaminants at all tested inoculum levels even the lowest $\left(10^{6} \mathrm{CFU} / \mathrm{mL}\right)$. This demonstrated the robustness of the strategy applied in this work. There are only few reports comparing the antifungal activity of bioprotective cultures in both challengetest and natural contamination in real products (shelf life test). A study on the antifungal activity of $2 \mathrm{~L}$. amylovorus strains used as adjunct cultures in miniature Cheddar cheese showed that the $10^{3}$ spores inoculated of $P$. expansum were inhibited for 1-4 days, while the airborne contaminants were inhibited for 6 days (Lynch et al., 2014). Some studies showed the effectiveness of LAB in situ against natural contaminants, e.g., L. amylovarus DSM1928 active in wheat, gluten-free and quinoa bread (Axel et al., 2015, 2016a,b), a semi-liquid biopreserver (SL778) developed from L. plantarum CRL 778 in bread (Gerez et al., 2015), and fermentates of $P$. jensenii and L. rhamnosus in sour cream and semi-hard cheeses (Ganier et al. submitted). 
Some of the constraints related to the potential use of bioprotective cultures (Leyva Salas et al., 2017) were also considered in this study, including safety, sensory and economic constraints (level of inoculation). From in vitro tests in dairy products to the scale up in sour cream and semi-hard cheese work, we designed the study to reduce the gap often observed between the antifungal activity in vitro and in situ. Concerning the safety of the strains to be used as adjunct cultures, although lactobacilli are not known as pathogens, it is necessary to follow a rigorous safety assessment procedure, taking multiple criteria into account. Recently, biogenic production and antibiotic resistance pattern was studied in some of the strains used in this study (Coton et al., 2018). In the latter study, L. brevis L128 was identified as a biogenic amine-producer, which precludes its use as a potential bioprotective culture, and led us to exclude the A2 and A4 combinations including this strain, despite its high antifungal activity. Beyond the latter activity, it is crucial to evaluate the impact of the adjunct cultures on the sensory properties of the treated dairy products. The sensory proximity of the creams with the reference culture $\mathrm{X} 1$ and the creams with A1 and A3 suggests that the selected combinations could also be used without a marked adverse impact on cream sensory properties. Concerning the more acid flavor reported by the judges in the creams containing A1 and A3, it could be linked to a post-acidification increase compared to the products without adjuncts. Similarly, some commercial antifungal cultures used as antifungal in dairy products are known to increase post-acidification, especially when the products are stored at ambient temperatures (Nielsen et al., 2017). Despite organoleptic differences and post-acidification at high inoculum levels, the dairy products containing the antifungal combinations were still acceptable by the judges.

The minimal efficient inoculum concentration is important from an economic point of view. An initial concentration of $10^{7} \mathrm{CFU} / \mathrm{mL}$ (i.e., $5.10^{6} \mathrm{CFU} / \mathrm{mL}$ of each strain) was efficient in both models from this work. In another study in yogurt, it was demonstrated that an inoculum level of $10^{5} \mathrm{CFU} / \mathrm{mL}$ of L. harbinensis K.V9.3.1Np was necessary to get an antifungal activity, but $5.10^{6} \mathrm{CFU} / \mathrm{mL}$ was required for a total inhibition (Delavenne et al., 2015). To explain the variation in the antifungal activity of A1 observed in sour cream at the lowest inoculation level, $10^{6} \mathrm{CFU} / \mathrm{mL}$, we can hypothesize that a minimal limit of population at the moment of contamination is required to express an inhibitory effect. A similar phenomena was reported in yogurt as to inhibit $Y$. lipolytica by L. harbinensis

\section{REFERENCES}

Aunsbjerg, S. D., Honoré, A. H., Marcussen, J., Ebrahimi, P., Vogensen, F. K., Benfeldt, C., et al. (2015). Contribution of volatiles to the antifungal effect of Lactobacillus paracasei in defined medium and yogurt. Int. J. Food Microbiol. 194, 46-53. doi: 10.1016/j.ijfoodmicro.2014.11.007

Axel, C., Brosnan, B., Zannini, E., Furey, A., Coffey, A., and Arendt, E. K. (2016a). Antifungal sourdough lactic acid bacteria as biopreservation tool in quinoa and rice bread. Int. J. Food Microbiol. 239, 86-94. doi: 10.1016/j.ijfoodmicro.2016.05.006
K.V9.3.1Np, 2.5-3.10 $\mathrm{CFU} / \mathrm{g}$ of L. harbinensis were necessary at the moment of contamination (Delavenne et al., 2015). The authors suggested an all-or-nothing mechanism (no inhibition below organic acid MICs and total inhibition above), and a quorum sensing regulatory mechanism as in bacteriocinproduction by LAB. The fact that, for a same antifungal culture, a variable antifungal activity occurred between semi-hard cheese assays also suggests a role of biotic and abiotic factors resulting from interactions between the food, the antifungal cultures and the fungal contaminants (Leyva Salas et al., 2017). The potential influencing factors include the dry matter content in cheeses, ripening chamber humidity, interactions between the smear and the fungi, and non-sterility of the ripening chambers.

This study presents an effective approach from in vitro screening to in situ application to develop antifungal combinations with high antifungal activity and broad spectrum, by covering different aspects that limit the entrance of bioprotective cultures to the market. The two selected binary combinations (i.e., A1 and A3) are good candidates for the antifungal biopreservation of dairy products.

\section{AUTHOR CONTRIBUTIONS}

EC, AT, JM, FV, and MLS designed the experiments. MLS and $\mathrm{ML}$ performed in vitro experiments. GG, $\mathrm{MH}-\mathrm{O}, \mathrm{MC}$, and MLS performed scale pilot experiments. SL contributed to the conception and data analysis of the sensory evaluations. MLS, AT, and EC wrote the manuscript. EC, AT, JM, and FV supervised the project. All authors discussed the results and contributed to the final manuscript.

\section{ACKNOWLEDGMENTS}

This work was financially supported by the Conseils Régionaux de Bretagne and des Pays de la Loire, France (grants $\mathrm{N}^{\circ} 13008651$ and $\mathrm{N}^{\circ}$ 2014-01081, respectively) in the framework of the PROFIL interregional project under the scientific coordination of INRA (J. Leonil) and managed by the Bretagne Biotechnologie Alimentaire (BBA) industrial association.

\section{SUPPLEMENTARY MATERIAL}

The Supplementary Material for this article can be found online at: https://www.frontiersin.org/articles/10.3389/fmicb. 2018.01787/full\#supplementary-material 
in rice cakes. J. Microbiol. 50, 842-848. doi: 10.1007/s12275-0122153-y

Batish, V. K., Roy, U., Lal, R., and Grower, S. (1997). Antifungal attributes of lactic acid bacteria - a review. Crit. Rev. Biotechnol. 17, 209-225. doi: 10.3109/07388559709146614

Bourdichon, F., Casaregola, S., Farrokh, C., Frisvad, J. C., Gerds, M. L., Hammes, W. P., et al. (2012). Food fermentations: microorganisms with technological beneficial use. Int. J. Food Microbiol. 154, 87-97. doi: 10.1016/j.ijfoodmicro.2011.12.030

Cheong, E. Y. L., Sandhu, A., Jayabalan, J., Kieu Le, T. T., Nhiep, N. T., My Ho, H. T., et al. (2014). Isolation of lactic acid bacteria with antifungal activity against the common cheese spoilage mould Penicillium commune and their potential as biopreservatives in cheese. Food Control 46, 91-97. doi: 10.1016/j.foodcont.2014.05.011

Coton, M., Lebreton, M., Leyva Salas, M., Garnier, L., Navarri, M., Pawtowski, A., et al. (2018). Biogenic amine and antibiotic resistance profiles determined for lactic acid bacteria and a propionibacterium prior to use as antifungal bioprotective cultures. Int. Dairy J. 85, 21-26. doi: 10.1016/j.idairyj.2018. 05.001

Crowley, S., Mahony, J., and van Sinderen, D. (2013). Current perspectives on antifungal lactic acid bacteria as natural bio-preservatives. Trends Food Sci. Technol. 33, 93-109. doi: 10.1016/j.tifs.2013.07.004

Delavenne, E., Cliquet, S., Trunet, C., Barbier, G., Mounier, J., and Le Blay, G. (2015). Characterization of the antifungal activity of Lactobacillus harbinensis K.V9.3.1Np and Lactobacillus rhamnosus K.C8.3.1I in yogurt. Food Microbiol. 45, 10-17. doi: 10.1016/j.fm.2014.04.017

Delavenne, E., Ismail, R., Pawtowski, A., Mounier, J., Barbier, G., and Le Blay, G. (2013). Assessment of lactobacilli strains as yogurt bioprotective cultures. Food Control 30, 206-213. doi: 10.1016/j.foodcont.2012.06.043

Delavenne, E., Mounier, J., Déniel, F., Barbier, G., and Le Blay, G. (2012). Biodiversity of antifungal lactic acid bacteria isolated from raw milk samples from cow, ewe and goat over one-year period. Int. J. Food Microbiol. 155, 185-190. doi: 10.1016/j.ijfoodmicro.2012.02.003

El-Shafei, K., Mam, A. E. G., Dabiza, N., Sharaf, O. M., and Effat, B. A. (2008). A mixed culture of Propionibacterium thoenii P-127, Lactobacillus rhamnosus and Lactobacillus plantarum as protective cultures in Kareish cheese. Pol. J. Food Nutr. Sci. 58, 433-441. Available online at: https://journal.pan.olsztyn.pl/ fd.php?f $=1095$

Fernandez, B., Vimont, A., Desfossés-Foucault, É., Daga, M., Arora, G., and Fliss, I. (2017). Antifungal activity of lactic and propionic acid bacteria and their potential as protective culture in cottage cheese. Food Control 78, 350-356. doi: 10.1016/j.foodcont.2017.03.007

Fröhlich-Wyder, M. T., Bisig, W., Guggisberg, D., Jakob, E., Turgay, M., and Wechsler, D. (2017). "Cheeses with propionic acid fermentation," in Cheese (Bern: Elsevier), 889-910.

Fuselli, F., Guarino, C., La Mantia, A., Longo, L., Faberi, A., and Marianella, R. M. (2012). Multi-detection of preservatives in cheeses by liquid chromatography-tandem mass spectrometry. J. Chromatogr. B 906, 9-18. doi: 10.1016/j.jchromb.2012.07.035

Garnier, L., Leyva Salas, M., Pinon, N., Wiernasz, N., Pawtowski, A., Coton, E., et al. (2018). Technical note: high-throughput method for antifungal activity screening in a cheese-mimicking model. J. Dairy Sci. 101, 4971-4976. doi: $10.3168 /$ jds.2017-13518

Garnier, L., Valence, F., Pawtowski, A., Auhustsinava-Galerne, L., Frotté, N., Baroncelli, R., et al. (2016). Diversity of spoilage fungi associated with various French dairy products. Int. J. Food Microbiol. 241, 191-197. doi: 10.1016/j.ijfoodmicro.2016.10.026

Gerez, C. L., Fornaguera, M. J., Obregozo, M. D., Font de Valdez, G., and Torino, M. I. (2015). Antifungal starter culture for packed bread: influence of two storage conditions. Rev. Argent. Microbiol. 47, 118-124. doi: 10.1016/j.ram.2015.02.002

Gómez-Torres, N., Ávila, M., Delgado, D., and Garde, S. (2016). Effect of reuterin-producing Lactobacillus reuteri coupled with glycerol on the volatile fraction, odour and aroma of semi-hard ewe milk cheese. Int. J. Food Microbiol. 232, 103-110. doi: 10.1016/j.ijfoodmicro.2016. 05.031
Hartemink, R., Domenech, V. R., and Rombouts, F. M. (1997). LAMVABa new selective medium for the isolation of Lactobacilli from faeces. J. Microbiol. Methods 29, 77-84. doi: 10.1016/S0167-7012(97) 00025-0

Hemme, D., and Foucaud-Scheunemann, C. (2004). Leuconostoc, characteristics, use in dairy technology and prospects in functional foods. Int. Dairy J. 14, 467-494. doi: 10.1016/j.idairyj.2003.10.005

Hornbaek, T., Lisberg, M., and Diemer, S. K. (2014). Synergistic Antimicrobial Effect. U.S. Patent Application No. 14/110,388 1. U.S. Patent and Trademark Office.

Inglin, R. C., Stevens, M. J. A., Meile, L., Lacroix, C., and Meile, L. (2015). High-throughput screening assays for antibacterial and antifungal activities of Lactobacillus species. J. Microbiol. Methods 114, 26-29. doi: 10.1016/j.mimet.2015.04.011

Ledenbach, L. H., and Marshall, R. T. (2009). "Microbiological spoilage of dairy products," in Compendium of the Microbiological Spoilage of Foods and Beverages, eds W. H. Sperber, and M. P. Doyle (New York, NY: Springer), 41-67.

Le Lay, C., Coton, E., Le Blay, G., Chobert, J. M., Haertlé, T., Choiset, Y., et al. (2016a). Identification and quantification of antifungal compounds produced by lactic acid bacteria and propionibacteria. Int. J. Food Microbiol. 239, 79-85. doi: 10.1016/j.ijfoodmicro.2016.06.020

Le Lay, C., Mounier, J., Vasseur, V., Weill, A., Le Blay, G., Barbier, G., et al. (2016b). In vitro and in situ screening of lactic acid bacteria and propionibacteria antifungal activities against bakery product spoilage molds. Food Control 60, 247-255. doi: 10.1016/j.foodcont.2015.07.034

Leroy, F., and De Vuyst, L. (2004). Lactic acid bacteria as functional starter cultures for the food fermentation industry. Trends Food Sci. Technol. 15, 67-78. doi: 10.1016/j.tifs.2003.09.004

Lê, S., and Worch, T. (2014). Analyzing Sensory Data with R, 1st Edn. Boca Raton, FL: Chapman and Hall/CRC Press.

Leyva Salas, M., Mounier, J., Valence, F., Coton, M., Thierry, A., and Coton, E. (2017). Antifungal microbial agents for food biopreservationa review. Microorganisms 5:E37. doi: 10.3390/microorganisms50 30037

Li, H., Liu, L., Zhang, S., Uluko, H., Cui, W., and Lv, J. (2013). Potential use of Lactobacillus casei AST18 as a bioprotective culture in yogurt. Food Control 34, 675-680. doi: 10.1016/j.foodcont.2013.06.023

Lynch, K. M., Pawlowska, A. M., Brosnan, B., Coffey, A., Zannini, E., Furey, A., et al. (2014). Application of Lactobacillus amylovorus as an antifungal adjunct to extend the shelf-life of Cheddar cheese. Int. Dairy J. 34, 167-173. doi: 10.1016/j.idairyj.2013.07.017

Martley, F. G., and Crow, V. L. (1993). Interactions between non-starter microorganisms during cheese manufacture and repening. Int. Dairy J. 3, 461-483. doi: 10.1016/0958-6946(93)90027-W

Ngang, J. J. E., Yadang, G., Kamdem, S. L. S., Kouebou, C. P., Fanche, S. A. Y., Kougan, D. L. T., et al. (2015). Antifungal properties of selected lactic acid bacteria and application in the biological control of ochratoxin A producing fungi during cocoa fermentation. Biocontrol Sci. Technol. 25, 245-259. doi: 10.1080/09583157.2014.969195

Nielsen, C. L. M., Hornbaek, T., Rasmussen, P., and Poulsen, L. (2017). Lactobacillus fermentum Bacteria Inhibiting Post-acidification. World Intellectual Property Organization Patent Application No. WO2017037052A1. U.S. Patent and Trademark Office.

Özcelik, S., Kuley, E., and Özogul, F. (2016). Formation of lactic, acetic, succinic, propionic, formic and butyric acid by lactic acid bacteria. LWT Food Sci. Technol. 73, 536-542. doi: 10.1016/j.lwt.2016.06.066

Pitt, J. I., and Hocking, A. D. (2009). Fungi and Food Spoilage. Boston, MA: Springer.

Russo, P., Arena, M. P., Fiocco, D., Capozzi, V., Drider, D., and Spano, G. (2017). Lactobacillus plantarum with broad antifungal activity: a promising approach to increase safety and shelf-life of cereal-based products. Int. J. Food Microbiol. 247, 48-54. doi: 10.1016/j.ijfoodmicro.2016.04.027

Salvat-Brunaud, D., Thierry, A., and Maubois, J. L. (1997). Development of a medium simulating emmental juice for propionibacteria growth. J. Dairy Res. 64, 573-580. doi: 10.1017/S0022029997002410 
Schwenninger, S. M., and Meile, L. (2004). A mixed culture of Propionibacterium jensenii and Lactobacillus paracasei subsp. paracasei inhibits food spoilage yeasts. Syst. Appl. Microbiol. 27, 229-237. doi: 10.1078/072320204322881853

Silva, M., and Lidon, F. (2016). Food preservatives - an overview on applications and side effects. Emir. J. Food Agric. 28, 366-373. doi: 10.9755/ejfa.201604-351

Stark, J., and Tan, H. S. (2003). "Natamycin," in Food Preservatives, eds N. J. Russell and G. W. Gould (Boston, MA: Springer), 179-195.

Stiles, M. E. (1996). Biopreservation by lactic acid bacteria. Antonie Van Leeuwenhoek 70, 331-345. doi: 10.1007/BF00395940

Thierry, A., Maillard, M. B., and Yvon, M. (2002). Conversion of LLeucine to isovaleric acid by Propionibacterium freudenreichii TL 34 and ITGP23. Appl. Environ. Microbiol. 68, 608-615. doi: 10.1128/AEM.68.2.608615.2002

Valerio, F., Favilla, M., De Bellis, P., Sisto, A., de Candia, S., and Lavermicocca, P. (2009). Antifungal activity of strains of lactic acid bacteria isolated from a semolina ecosystem against Penicillium roqueforti, Aspergillus niger and
Endomyces fibuliger contaminating bakery products. Syst. Appl. Microbiol. 32, 438-448. doi: 10.1016/j.syapm.2009.01.004

Conflict of Interest Statement: The authors declare that the research was conducted in the absence of any commercial or financial relationships that could be construed as a potential conflict of interest.

The reviewer GT and handling Editor declared their shared affiliation at the time of the review.

Copyright (c) 2018 Leyva Salas, Thierry, Lemaitre, Garric, Harel-Oger, Chatel, Lê, Mounier, Valence and Coton. This is an open-access article distributed under the terms of the Creative Commons Attribution License (CC BY). The use, distribution or reproduction in other forums is permitted, provided the original author(s) and the copyright owner(s) are credited and that the original publication in this journal is cited, in accordance with accepted academic practice. No use, distribution or reproduction is permitted which does not comply with these terms. 